\title{
Implications of ANEC for SCFTs in four dimensions
}

\author{
Andrea Manenti, ${ }^{a, b}$ Andreas Stergiou $^{c}$ and Alessandro Vichi ${ }^{a}$ \\ ${ }^{a}$ Institute of Physics, École Polytechnique Fédérale de Lausanne, \\ CH-1015 Lausanne, Switzerland \\ ${ }^{b}$ Simons Center for Geometry and Physics, \\ Stony Brook, NY 11794, U.S.A. \\ ${ }^{c}$ Theoretical Division, MS B285, Los Alamos National Laboratory, \\ Los Alamos, NM 87545, U.S.A. \\ E-mail: andrea.manenti@epfl.ch, andreas@lanl.gov, \\ alessandro.vichi@unipi.it
}

ABStRaCt: We explore consequences of the Averaged Null Energy Condition (ANEC) for scaling dimensions $\Delta$ of operators in four-dimensional $\mathcal{N}=1$ superconformal field theories. We show that in many cases the ANEC bounds are stronger than the corresponding unitarity bounds on $\Delta$. We analyze in detail chiral operators in the $\left(\frac{1}{2} j, 0\right)$ Lorentz representation and prove that the ANEC implies the lower bound $\Delta \geqslant \frac{3}{2} j$, which is stronger than the corresponding unitarity bound for $j>1$. We also derive ANEC bounds on $\left(\frac{1}{2} j, 0\right)$ operators obeying other possible shortening conditions, as well as general $\left(\frac{1}{2} j, 0\right)$ operators not obeying any shortening condition. In both cases we find that they are typically stronger than the corresponding unitarity bounds. Finally, we elucidate operator-dimension constraints that follow from our $\mathcal{N}=1$ results for multiplets of $\mathcal{N}=2,4$ superconformal theories in four dimensions. By recasting the ANEC as a convex optimization problem and using standard semidefinite programming methods we are able to improve on previous analyses in the literature pertaining to the nonsupersymmetric case.

Keywords: Conformal Field Theory, Superspaces

ARXiv EPrint: 1905.09293 


\section{Contents}

1 Introduction and summary of results 1

1.1 Strategy 2

1.2 Summary of results 3

2 Setup 6

3 Constraints on the supersymmetric three-point correlator 9

3.1 Conservation 9

3.2 Reality 9

$\begin{array}{lll}3.3 & \text { Ward identities } & 10\end{array}$

$\begin{array}{lll}3.4 & \text { Shortening conditions } & 13\end{array}$

4 Expansion of the superspace correlator $\quad 14$

$\begin{array}{lll}4.1 & \text { Lowest order } & 15\end{array}$

$\begin{array}{lll}4.2 & \text { Three-point function }\langle\bar{O} T O\rangle & 15\end{array}$

4.3 Three-point functions $\langle(\bar{Q} \bar{O}) S O\rangle$ and $\langle(Q \bar{O}) \bar{S} O\rangle \quad 16$

4.4 Three-point functions $\langle(\bar{Q} \bar{O}) J(Q O)\rangle$ and $\langle(Q \bar{O}) J(\bar{Q} O)\rangle \quad 16$

4.5 Three-point functions $\langle(\bar{Q} \bar{O}) T(Q O)\rangle$ and $\langle(Q \bar{O}) T(\bar{Q} O)\rangle \quad 16$

$\begin{array}{lll}5 & \text { The averaged null energy condition } & 17\end{array}$

$\begin{array}{lll}5.1 & \text { Operators of spin }\left(\frac{1}{2} j, 0\right) & 17\end{array}$

$\begin{array}{lll}5.2 & \text { ANEC on a superposition of states } & 18\end{array}$

$\begin{array}{ll}5.3 \text { Operators of } \operatorname{spin}\left(\frac{1}{2} j, \frac{1}{2}\right) & 19\end{array}$

5.4 The ANEC as a semidefinite programming problem 20

5.5 Details on ANEC bounds: nonsupersymmetric case 21

5.6 Details on ANEC bounds: supersymmetric case 22

6 Bounds on extended supersymmetry multiplets 24

6.1 Conventions 24

$6.2 \mathcal{N}=2 \quad 24$

$\begin{array}{ll}6.3 \mathcal{N}=4 & 25\end{array}$

7 Conclusions and outlook $\quad 26$

$\begin{array}{ll}\text { A Supersymmetric inversion tensors } & 27\end{array}$

B Proof of the general formula 28

B.1 Formula for the $\left(\frac{1}{2} j, 0\right)$ case 28

B.2 Formula for the $\left(\frac{1}{2} j, \frac{1}{2}\right)$ case 29

C Tables $\quad 31$

C.1 Ward identities 31

C.2 Expansion in components 33 


\section{Introduction and summary of results}

In recent years attention has been brought to the utility of expectation values of integrated projections of the stress-energy tensor along null lines in conformal field theories (CFTs). Such observables have a long history in jet physics — see for example [1-3] — and they were first examined in the CFT context in the seminal work [4]. There, it was shown that an energy-positivity condition implies constraints on the coefficients in the three-point function of the stress-energy tensor. More precisely, given a state $|\psi\rangle$ of a local CFT with stress-energy tensor $T_{\mu \nu}$ and a null geodesic parametrized by $\lambda$ with tangent vector $u^{\mu}$, the following inequality, called the Averaged Null Energy Condition (ANEC), holds:

$$
\langle\psi|\mathcal{E}| \psi\rangle=\int_{-\infty}^{\infty} \mathrm{d} \lambda\left\langle\psi\left|T_{\mu \nu}\right| \psi\right\rangle u^{\mu} u^{\nu} \geqslant 0
$$

In [4] this was viewed as a positivity requirement for the energy measured by a hypothetical "calorimeter" placed at a large distance from the region where $|\psi\rangle$ is localized. The inequality (1.1) was later established more rigorously as a theorem $[5,6]$. It has also been understood that the ANEC is part of a larger class of constraints, which also follow from a thought collider experiment, namely the deep inelastic scattering bounds [7, 8], which state the positivity of an expectation value similar to (1.1) but with $T_{\mu \nu}$ replaced by the lowest-twist operator of a given spin $\ell>2$. Recently it was shown that the integral (1.1) is a special case of a larger set of integral transforms [9].

An important, perhaps unexpected application of (1.1) is that it implies lower bounds on operator dimensions $\Delta$ in CFTs [10]. It is of course known that in CFTs scaling dimensions of operators are bounded from below as a consequence of unitarity $[11,12]$. This is true independently of locality properties of the CFT, i.e. it does not rely on the presence of a stress-energy tensor in the CFT spectrum. However, it was demonstrated in [10] that in CFTs with a stress-energy tensor the unitarity bound is suboptimal for some classes of operators. The analysis of a few examples led [10] to the conjecture $\Delta \geqslant \max \{j, \bar{\jmath}\}$, where $\left(\frac{1}{2} j, \frac{1}{2} \bar{\jmath}\right)$ is the Lorentz representation of the CFT operator. This becomes stronger than the unitarity bound whenever $|j-\bar{\jmath}|>4$. We find that this conjecture is not supported by the ANEC for large values of $j$ in the case of $\left(\frac{1}{2} j, 0\right)$ and $\left(\frac{1}{2} j, \frac{1}{2}\right)$ operators - see figures 5 and 6 below.

In this work we apply the methods of [10] to four-dimensional $\mathcal{N}=1$ superconformal field theories (SCFTs). We find that for certain classes of operators the unitarity bounds of [13-15] cannot be saturated. Just as in [10], our results follow from a careful analysis of three-point functions of the schematic type $\left\langle\bar{O} T_{\mu \nu} O\right\rangle$ with $O$ a conformal primary and $\bar{O}$ its conjugate. The difference with the nonsupersymmetric case is that here such conformal three-point functions are encoded in superconformal three-point functions involving the Ferrara-Zumino multiplet [16]. The constraints of $4 \mathrm{~d} \mathcal{N}=1$ superconformal symmetry on three-point functions of superconformal primary operators have been examined in great detail in $[17,18]$, and we rely heavily on those results.

The constraints imposed by the ANEC and explored in [10] are schematically of the form

$$
\Delta_{O}>\Delta_{\mathrm{ANEC}}(j, \bar{\jmath}) \text { and } M\left(\lambda_{O \bar{O} T}, \Delta_{O}\right) \succeq 0
$$


where $M$ is a matrix that depends linearly on the three-point function coefficients $\lambda_{O \bar{O} T}$. In a nonsupersymmetric theory, the constraints on the three-point function coefficients generically admit a solution. Therefore, the first condition determines the bound on operator dimensions.

In the presence of supersymmetry things can change significantly. First, there exist certain multiplet shortening conditions, without a nonsupersymmetric analog, that fix some of the three-point function coefficients $\lambda_{O \bar{O} T}$, thus leaving less freedom to satisfy (1.2). Moreover, even without imposing any shortening conditions, the ANEC must hold on any state $|\psi\rangle$ given by the most general superposition of states in a super-multiplet schematically

$$
|\psi\rangle \sim(O+\alpha Q O+\beta \bar{Q} O+\ldots)|0\rangle .
$$

Computing the integral (1.1) on states (1.3) leads to more intricate constraints on the threepoint function coefficients $\lambda_{O \bar{O} T}$, which sometimes do not admit a solution. Intuitively, we then expect that in the presence of supersymmetry a broader class of operators will violate the ANEC and must thus be absent in any unitary local SCFT.

In the remainder of this section we briefly outline the logic behind our computations and present our final results. The rest of the paper carefully goes through the details of our calculations.

\section{$1.1 \quad$ Strategy}

In this work we focus on superconformal multiplets $\mathcal{O}(x, \theta, \bar{\theta})$ for which the lowest component field $O$ transforms in the $\left(\frac{1}{2} j, 0\right)$ irreducible representation of the Lorentz group. Our first goal is to determine the most general form of the three-point function in superspace among $\mathcal{O}$, its complex conjugate superfield, and the Ferrara-Zumino multiplet $\mathcal{J}$, which contains the stress-energy tensor: ${ }^{1}$

$$
\left\langle\overline{\mathcal{O}}\left(\mathbf{z}_{1}\right) \mathcal{J}\left(\mathbf{z}_{2}\right) \mathcal{O}\left(\mathbf{z}_{3}\right)\right\rangle, \quad \mathbf{z}_{i}=\left(x_{i}, \theta_{i}, \bar{\theta}_{i}\right) .
$$

In order to determine (1.4), in section 2 we write the most general three-point function consistent with $\mathcal{N}=1$ superconformal invariance, complex conjugation, and conservation of the Ferrara-Zumino multiplet. Next, we fix certain combinations of the three-point function coefficients entering (1.4) by imposing the Ward identities generated by the conserved currents $J_{R}^{\mu}, T_{\mu \nu}$ and $S_{\mu}^{\alpha}$ in $\mathcal{J}$. Although in principle it should be possible to obtain a superspace version of the Ward identities, along the lines of [18], in this work we impose the constraints at the level of the individual primaries and superdescendants. More specifically, we find that once the $J_{\mu}^{R}$ and $T_{\mu \nu}$ Ward identities are imposed in the three-point function involving the superprimary $O$, all other ones we checked follow. ${ }^{2}$

In addition to the above, the three-point function (1.4) could satisfy further constraints, associated to various shortening conditions of the superconformal multiplet $\mathcal{O}$. Following

\footnotetext{
${ }^{1}$ In this section we only present schematic formulas. Details are given in the next sections.

${ }^{2}$ More specifically, we checked the Ward identities for $\left\langle(\bar{Q} \bar{O}) J_{R}(Q O)\right\rangle,\langle(\bar{Q} \bar{O}) T(Q O)\rangle$ and $\langle(\bar{Q} \bar{O}) S O\rangle$. In principle there could be extra relations that we did not take into account.
} 
the convention of [19] we denote $\mathcal{N}=1$ multiplets as $\left[\mathcal{X}_{L}, \overline{\mathcal{X}}_{R}\right]$, where $\mathcal{X}_{L, R}$ represents the action of the charges $Q$ and $\bar{Q}$, which give rise to long $(L)$, semi-short $\left(A_{1}, A_{2}\right)$ or chiral $(B)$ multiplets. We spell out the exact definitions in section 3.4, together with the corresponding unitarity bounds, and we compute the most general form of (1.4) compatible with these constraints. The results are summarized in the tables in appendix C.1.

As a final step, we need to decompose the superspace three-point function in the various $\theta$ components and extract the nonsupersymmetric three-point functions of the superprimary $O$ and various primary superdescendants. This task is performed in section 4 and summarized in the tables in appendix C.2. Unfortunately the computations required to perform this step become rapidly very complicated. In this work we only pushed to the fourth order in $\theta_{i}$ or $\bar{\theta}_{i}$ and computed three-point functions involving at most $T_{\mu \nu}$ and superdescendants $Q O^{ \pm}$and $\bar{Q} O .^{3}$

After all these preparatory steps, we can impose the ANEC (1.1) on a general state of the form of (1.3). Due to R-charge conservation, only a few three-point functions are non vanishing. In the end we impose that ${ }^{4}$

$$
\begin{gathered}
\langle O|\mathcal{E}| O\rangle \geqslant 0, \quad\langle(\bar{Q} O)|\mathcal{E}|(\bar{Q} O)\rangle \geqslant 0 \\
\text { and } \quad\left(\begin{array}{l}
\left\langle\left(Q O^{+}\right)|\mathcal{E}|\left(Q O^{+}\right)\right\rangle\left\langle\left(Q O^{+}\right)|\mathcal{E}|\left(Q O^{-}\right)\right\rangle \\
\left\langle\left(Q O^{-}\right)|\mathcal{E}|\left(Q O^{+}\right)\right\rangle\left\langle\left(Q O^{-}\right)|\mathcal{E}|\left(Q O^{-}\right)\right\rangle
\end{array}\right) \succeq 0 .
\end{gathered}
$$

We should stress that the above conditions are a subset of all conditions one can impose, since they do not include superdescendants of the form $Q^{2} O$ or $Q \bar{Q} O$ for example. Nevertheless, we find that in any unitary and local SCFT superprimaries that transform in the $\left(\frac{1}{2} j, 0\right)$ representation and satisfy the usual unitarity bounds do not necessarily satisfy the conditions (1.5).

In section 5 we obtain closed-form expressions for all the correlators appearing in (1.5) as rational functions of the spin $j$ and dimension $\Delta$. Such formulas allow us to easily compute bounds up to large values of $j$ and in some cases rigorously prove bounds for any $j$.

Finally, we explore the consequences of our analysis for theories with extended supersymmetry. In section 6 we consider special $\mathcal{N}=2$ and $\mathcal{N}=4$ supermultiplets and decompose them with respect to an $\mathcal{N}=1$ subalgebra. The ANEC constraints presented in the next subsection are then recast as bounds on the $\mathcal{N}=2,4$ superprimaries.

\subsection{Summary of results}

Let us first mention the results of our analysis for nonsupersymmetric CFTs, in the case of a conformal primary with dimension $\Delta$, transforming in $\left(\frac{1}{2} j, \frac{1}{2} \bar{\jmath}\right)$ representation, with

\footnotetext{
${ }^{3}$ We remind that the action of a supercharge produces in general two distinct primary superdescendants, which we schematically denote with \pm , with equal dimension and R-charge but transforming in different Lorentz representations. For operators in the $\left(\frac{1}{2} j, 0\right)$ representation only $\bar{Q} O^{+}$exists, so we will denote it as $\bar{Q} O$.

${ }^{4}$ For certain short supermultiplets some of these three-point functions vanish.
} 
$\bar{\jmath}=0,1$. In section 5.5 we show convincing evidence that the ANEC requires

$$
\Delta \geqslant \min \left(j, \frac{1}{15}(13 j+42)\right) \text {. }
$$

For $\bar{\jmath}=0,1$ the above expression is stronger than the corresponding unitarity bound for $j>2,6$, respectively. Although we don't have an analytic proof, we checked (1.6) up to $j=10^{3}$.

Next, we summarize the results of applying the ANEC inequality to superconformal multiplets $\mathcal{O}^{(j, 0)}$. We present them as bounds on the quantum numbers $q, \bar{q}$, which are related to the dimension and the R-charge of a given operator by the simple relations

$$
\Delta=q+\bar{q}, \quad R=\frac{2}{3}(q-\bar{q}) .
$$

We considered all possible shortening conditions classified in [19] and we also follow their notation, ${ }^{5}$ which we briefly explain in section 3.4 .

All cases for $\boldsymbol{j}=\mathbf{0}$. In this case the ANEC requires only $q \geqslant 0$ and $\bar{q} \geqslant 0$. Therefore, it is never stronger than the unitarity bound.

$\left[A_{1}, \bar{B}\right]$ for $j=1$. For these operators there are no free three-point function coefficients and the dimension and R-charge are fixed. It can be easily verified that the ANEC holds.

$\left[\boldsymbol{A}_{\mathbf{1}}, \overline{\boldsymbol{B}}\right]$ for $\boldsymbol{j} \geqslant \mathbf{2}$. As shown in table 1 , these operators do not admit a three-point function with the Ferrara-Zumino multiplet consistent with all conditions. They are therefore absent in any local SCFT. ${ }^{6}$

$[\boldsymbol{L}, \overline{\boldsymbol{B}}]$ for $\boldsymbol{j} \geqslant \mathbf{1}$. With this shortening condition, corresponding to chiral operators, there are no free three-point function coefficients. Therefore the ANEC for any given $j$ is simply a system of inequalities on $q$ that can be solved algebraically. The result is

$$
\Delta=q \geqslant \frac{3}{2} j .
$$

This is equivalent to the unitarity bound for $j=1$ and it is stronger for all $j>1$. This result is not in contradiction with already known Lagrangian constructions, which so far have only provided examples for $j=1[20,21]$. Also note that the bound is saturated by $j$ copies of a free $j=1$ superconformal chiral primary $\psi_{i}^{\alpha}$

$$
\Psi^{\alpha_{1} \ldots \alpha_{j}}=: \psi_{1}^{\left(\alpha_{1}\right.} \cdots \psi_{j}^{\left.\alpha_{j}\right)}:
$$

In $\mathcal{N}=2$ theories, the bound in (1.8) implies a constraint on the dimension of the so called "exotic chiral primaries" discussed in [22]. In section 6.2 we show that

$$
\Delta_{\text {exotic }} \geqslant \frac{3}{2} j+1 \text {. }
$$

\footnotetext{
${ }^{5}$ In a nutshell, $L$ (resp. $\bar{L}$ ) stand for long, $B$ (resp. $\bar{B}$ ) for short or chiral, $A$ (resp. $\bar{A}$ ) for semi-short with respect to the supercharge $Q$ (resp. $\bar{Q})$.

${ }^{6}$ This conclusion does not require the ANEC.
} 


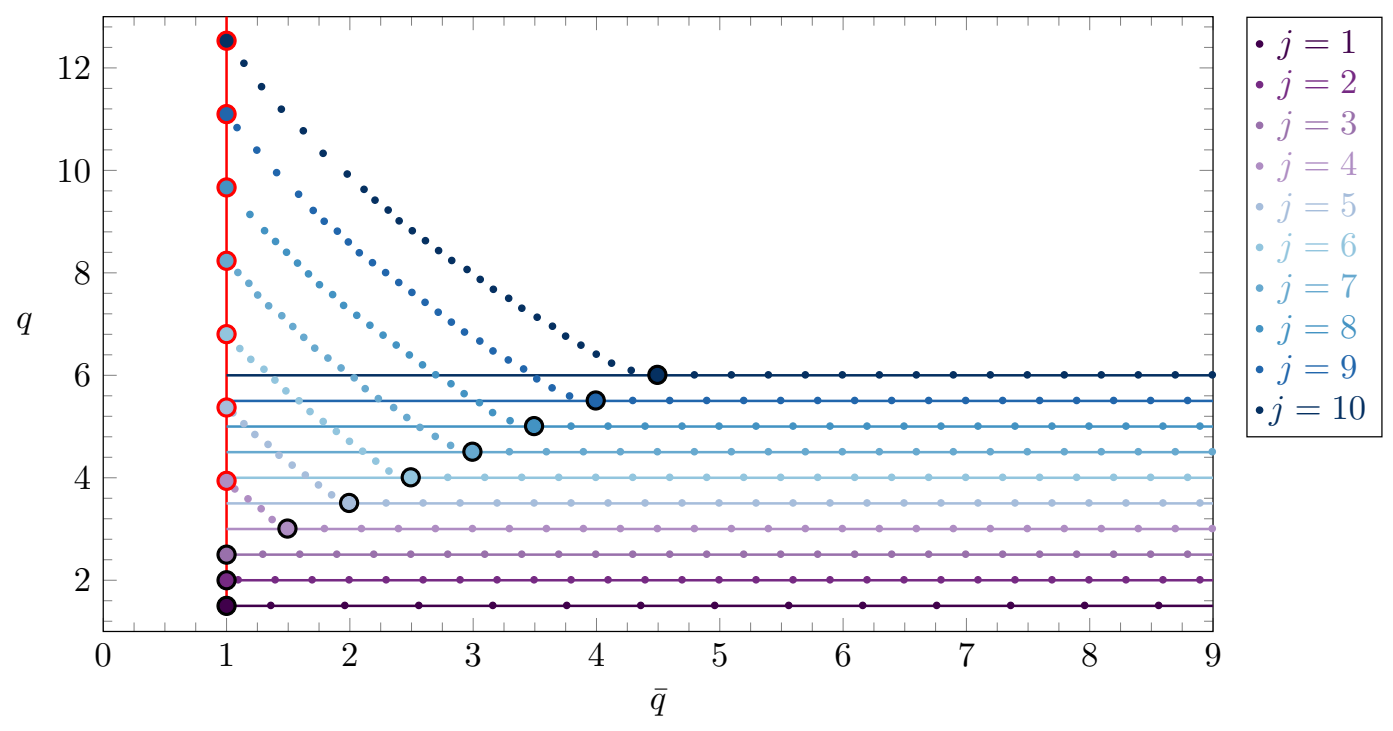

Figure 1. Lower bounds on the conformal dimension as a result of the ANEC for long multiples. Each point is the result of a bisection algorithm done with sdpb [23] (see section 5.4). The solid lines are the unitarity bound: the red line is the bound on $\bar{q}$ and the colored lines are the $j$-dependent bounds on $q$. The larger dots correspond to the points with shortening conditions $\left[L, \bar{A}_{2}\right]$ (for the red circled dots) and $\left[A_{1}, \bar{L}\right]$ (for the black circled dots).

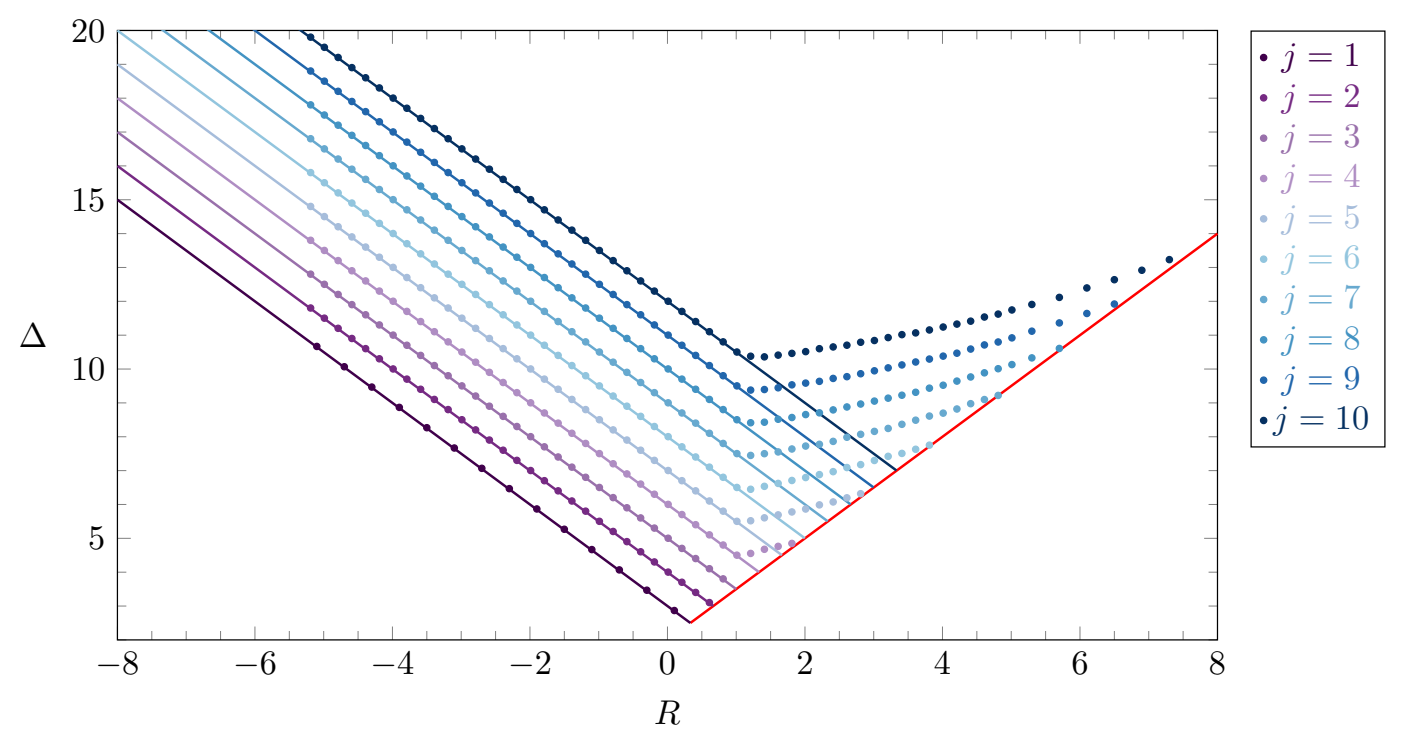

Figure 2. Plot of the results in figure 1 in the $(R, \Delta)$ plane.

$[\boldsymbol{L}, \overline{\boldsymbol{L}}]$ for $\boldsymbol{j} \geqslant \mathbf{1}$. In this case there are two free parameters $q$ and $\bar{q}$ and two undetermined three-point function coefficients (one for $j=1$ ). For every value of $j$ we fixed $\bar{q}$ and ran a bisection algorithm on $q$. The results are in figure 1 . See also figure 2 for a plot in the $(R, \Delta)$ plane.

$\left[\boldsymbol{L}, \overline{\boldsymbol{A}}_{\mathbf{2}}\right]$ for $\boldsymbol{j} \geqslant \mathbf{1}$. For this shortening condition the constraints are equivalent to $[L, \bar{L}]$ for $\bar{q}=1$. The results are given in figure 3 and correspond to the red circled dots on 


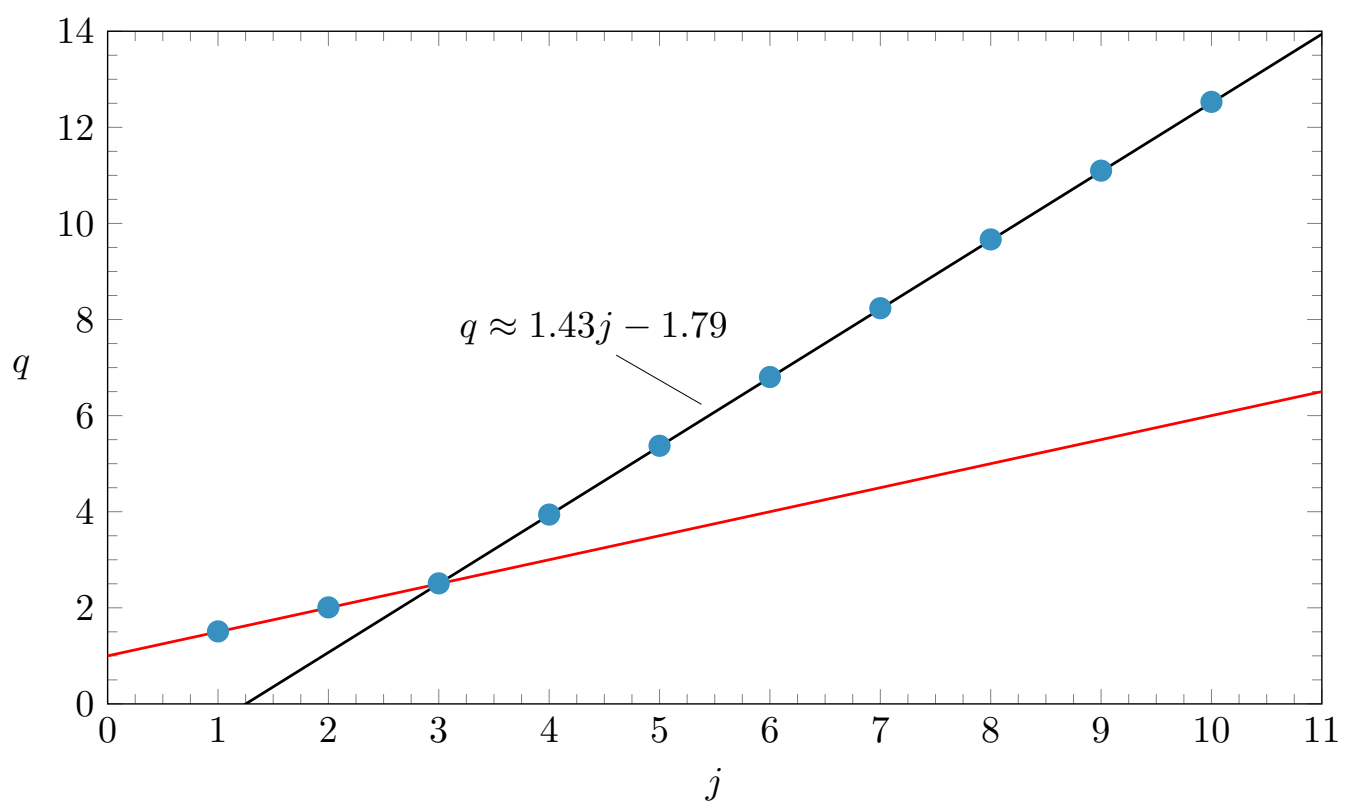

Figure 3. Lower bounds on the conformal dimension as a result of the ANEC for $\left[L, \bar{A}_{2}\right]$ multiplets. Each point is the result of a bisection algorithm done with sdpb [23] (see section 5.4). The red line is the unitarity bound $q=\frac{1}{2} j+1$. The operators for $j \leqslant 3$ that lie on the red line satisfy $\left[A_{1}, \bar{A}_{2}\right]$.

figure 1 . The operators at the unitarity bound, which satisfy the $\left[A_{1}, \bar{A}_{2}\right]$ shortening, are not allowed for $j>3$ (see below). Therefore, for $j>3$ the ANEC provides a constraint strictly stronger than unitarity.

$\left[\boldsymbol{A}_{1}, \bar{L}\right]$ for $j \geqslant 1$. Since for this case there is only one free three-point function coefficient and one parameter, $\bar{q}$, the system of inequalities is considerably simpler to solve. The results are given in figure 4 and correspond to the black circled dots on figure 1. As before, for $j>3$, the ANEC is strictly stronger than unitarity.

$\left[\boldsymbol{A}_{\mathbf{1}}, \overline{\boldsymbol{A}}_{\mathbf{2}}\right]$ for $\boldsymbol{j} \geqslant \mathbf{1}$. This condition admits solutions only for $j \leqslant 3$. In the edge case $j=3$ the ANEC inequalities fix the only independent three-point function coefficient to

$$
\mathcal{C}_{6}=-\frac{16}{\pi^{2}} \text {. }
$$

For all $j>3$ the ANEC admits no solution and thus such operators must be absent in any local SCFT.

\section{Setup}

Our object of study will be the three-point correlator in four dimensional $\mathcal{N}=1$ superspace of a superconformal multiplet $\mathcal{O}^{(j, 0)}(\mathbf{z})$, its conjugate $\overline{\mathcal{O}}^{(0, j)}(\mathbf{z})$ and the Ferrara-Zumino multiplet $\mathcal{J}^{(1,1)}(\mathbf{z})$. The charges of $\mathcal{J}$ are $q_{\mathcal{J}}=\bar{q}_{\mathcal{J}}=\frac{3}{2}$, while those of $\mathcal{O}$ and $\overline{\mathcal{O}}$ are $q_{\mathcal{O}}=\bar{q}_{\overline{\mathcal{O}}}=q$ and $q_{\overline{\mathcal{O}}}=\bar{q}_{\mathcal{O}}=\bar{q}$. The superscript $(j, \bar{\jmath})$ refers to the $\mathrm{SO}(3,1)$ representation, ${ }^{7}$

\footnotetext{
${ }^{7}$ Following standard conventions we denote the irreducible representations of the Lorentz group by $\left(\frac{1}{2} j, \frac{1}{2} \bar{\jmath}\right)$, where $j$ is the number of undotted and $\bar{\jmath}$ the number of dotted indices.
} 


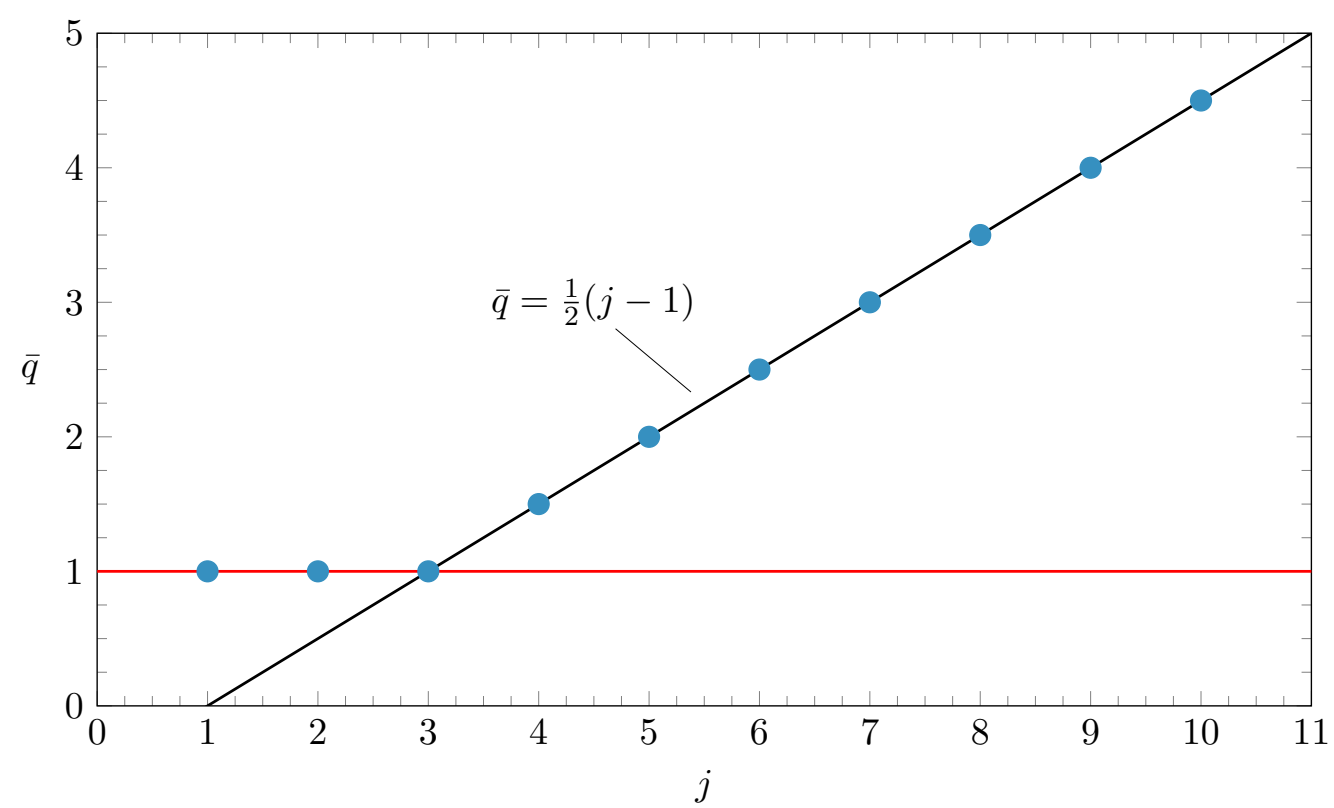

Figure 4. Lower bounds on the conformal dimension as a result of the ANEC for $\left[A_{1}, \bar{L}\right]$ multiplets. Each point is the result of a bisection algorithm done with Mathematica. The operators for $j \leqslant 3$ that lie on the red line satisfy $\left[A_{1}, \bar{A}_{2}\right]$.

and will be henceforth dropped for brevity. The shorthand $\mathbf{z}$ denotes

$$
\mathbf{z}_{i}=\left(z_{i}, \eta_{i}, \bar{\eta}_{i}\right), \quad \text { where } z_{i}=\left(x_{i}, \theta_{i}, \bar{\theta}_{i}\right) .
$$

The polarizations $\eta_{i}, \bar{\eta}_{i}$ are commuting spinors used to contract all free indices as follows:

$$
\mathcal{O}^{(j, \bar{\jmath})}(\mathbf{z})=\eta^{\alpha_{1}} \cdots \eta^{\alpha_{j}} \bar{\eta}^{\dot{\alpha}_{1}} \cdots \bar{\eta}^{\dot{\alpha}_{\bar{\jmath}}} \mathcal{O}_{\alpha_{1} \ldots \alpha_{j} \dot{\alpha}_{1} \ldots \dot{\alpha}_{\bar{\jmath}}}(z) .
$$

The tensor can be recovered by using spinor derivatives $\partial_{\eta}, \partial_{\bar{\eta}}$ which obey $\partial_{\eta^{\alpha}} \eta^{\beta}=\delta_{\alpha}^{\beta}$ and $\partial_{\eta^{\alpha}} \eta_{\beta}=\epsilon_{\beta \alpha}$, and similarly for the barred counterparts. We will follow the notation of [24] and the formalism introduced in [18].

The most general three-point function involving $\mathcal{O}^{(j, 0)}(\mathbf{z})$ can be written as

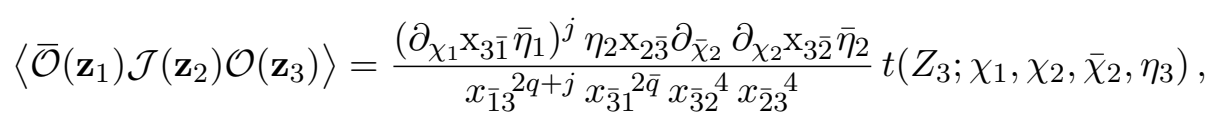

where $Z_{3}=\left(X_{3}, \Theta_{3}, \bar{\Theta}_{3}\right)$ will be defined shortly and $\chi_{i}, \bar{\chi}_{i}$ are auxiliary spinor polarizations. ${ }^{8}$ The function $t$ can be expressed as a linear combination of ten tensor structures, but the coefficients multiplying them are not arbitrary as they are constrained by reality of the correlator, conservation of $\mathcal{J}$, eventual shortening conditions on $\mathcal{O}$ and the Ward identities for the R-symmetry and the conformal group. We will analyze all these constraints in the next section. Let us now briefly describe all the fundamental building blocks of such tensor structures. They are functions of the supersymmetric interval $\mathrm{x}_{i \bar{\jmath}}$ defined as

$$
\left(\mathrm{x}_{i \bar{\jmath}}\right)_{\alpha \dot{\alpha}}=-\epsilon_{\alpha \beta} \epsilon_{\dot{\alpha} \dot{\beta}}\left(\tilde{\mathrm{x}}_{\bar{\jmath} i}\right)^{\dot{\beta} \beta}=-\sigma_{\alpha \dot{\alpha}}^{\mu}\left(x_{\bar{\jmath} i}\right)_{\mu}=\left(\mathrm{x}_{i j}\right)_{\alpha \dot{\alpha}}-2 i \theta_{i \alpha} \bar{\theta}_{i \dot{\alpha}}-2 i \theta_{j \alpha} \bar{\theta}_{j \dot{\alpha}}+4 i \theta_{i \alpha} \bar{\theta}_{j \dot{\alpha}}
$$

\footnotetext{
${ }^{8}$ They are denoted with a different letter than $\eta$ only to emphasize the fact that they are eventually removed by the derivatives in the numerator.
} 
with $x_{i j}=x_{i}-x_{j}, x_{\bar{\imath} j}{ }^{a}=\left(x_{\bar{\imath} j}{ }^{2}\right)^{a / 2}$ and of the Grassmann intervals $\theta_{i j}=\theta_{i}-\theta_{j}, \bar{\theta}_{i j}=\bar{\theta}_{i}-\bar{\theta}_{j}$. We can use these objects to define

$$
\begin{array}{ll}
\mathrm{X}_{3}=\frac{\mathrm{x}_{3 \overline{1}} \tilde{\mathrm{x}}_{\overline{1} 2} \mathrm{x}_{2 \overline{3}}}{x_{\overline{1} 3}{ }^{2} x_{\overline{3}}{ }^{2}}, & \overline{\mathrm{X}}_{3}=-\frac{\mathrm{x}_{3 \overline{2}} \tilde{\mathrm{x}}_{\overline{2} 1} \mathrm{x}_{1 \overline{3}}}{x_{\overline{3} 1}{ }^{2} x_{\overline{2}}{ }^{2}}=\mathrm{X}_{3}^{\dagger}, \\
\Theta_{3}=i\left(\frac{\mathrm{x}_{3 \overline{1}} \bar{\theta}_{31}}{x_{\overline{1}}{ }^{2}}-\frac{\mathrm{x}_{3 \overline{2}} \bar{\theta}_{32}}{x_{\overline{2}}{ }^{2}}\right), & \bar{\Theta}_{3}=i\left(\frac{\theta_{31} \mathrm{x}_{1 \overline{3}}}{x_{\overline{3} 1}^{2}}-\frac{\theta_{32} \mathrm{x}_{2 \overline{3}}}{x_{\overline{3} 2}{ }^{2}}\right)=\Theta_{3}^{\dagger} .
\end{array}
$$

Similar objects $\mathrm{X}_{i}, \Theta_{i}, \bar{\Theta}_{i}, i=1,2$, can be defined by a cyclic permutation of the points. We will further define

$$
\mathrm{U}_{3}=\frac{1}{2}\left(\mathrm{X}_{3}+\overline{\mathrm{X}}_{3}\right)
$$

Also, note that $\mathrm{X}_{3}-\overline{\mathrm{X}}_{3}=4 i \Theta_{3} \bar{\Theta}_{3}$. We can then form fully contracted monomials of the quantities defined above to obtain the building blocks of the tensor structures in $t$. A complete list is

$$
\begin{aligned}
& {[i \bar{j}]=\frac{\eta_{i} \mathrm{U} \bar{\eta}_{j}}{|U|}, \quad[\Theta \bar{\Theta}]=\frac{\Theta \mathrm{U} \bar{\Theta}}{U^{2}}, \quad[i j]=\eta_{i} \eta_{j}, \quad[\bar{\imath} \bar{j}]=\bar{\eta}_{i} \bar{\eta}_{j}, \quad\left[\Theta^{2}\right]=\frac{\Theta^{2}}{|U|},} \\
& {\left[\bar{\Theta}^{2}\right]=\frac{\bar{\Theta}^{2}}{|U|}, \quad[\Theta j]=\frac{\Theta \eta_{j}}{|U|^{1 / 2}}, \quad[\bar{\Theta} \bar{\jmath}]=\frac{\bar{\Theta} \bar{\eta}_{j}}{|U|^{1 / 2}}, \quad[j \bar{\Theta}]=\frac{\eta_{i} \mathrm{U} \bar{\Theta}}{|U|^{3 / 2}}, \quad[\Theta \bar{\jmath}]=\frac{\Theta \mathrm{U} \bar{\eta}_{j}}{|U|^{3 / 2}} .}
\end{aligned}
$$

Other than the physical constraints mentioned before, that will be addressed later, $t$ must satisfy certain homogeneity properties, which can be summarized as

$$
t\left(\lambda \bar{\lambda} X, \lambda \Theta, \bar{\lambda} \bar{\Theta} ; \kappa \eta_{1}, \mu \eta_{2}, \bar{\mu} \bar{\eta}_{2}, \bar{\kappa} \eta_{3}\right)=(\lambda \bar{\lambda})^{-3}(\kappa \bar{\kappa})^{j} \mu \bar{\mu} t\left(X, \Theta, \bar{\Theta} ; \eta_{i}, \bar{\eta}_{i}\right) .
$$

All possible tensor structures are built out of the blocks in eq. (2.7) times a factor $U^{-3}$ to take care of the $\lambda \bar{\lambda}$ scaling. Not all combinations will be independent due to several relations termed Schouten identities which stem from the vanishing of $\epsilon^{\alpha[\beta} \epsilon^{\gamma \delta]}$ and the corresponding tensor with dotted indices. We will now produce a list of ten tensor structures that we have explicitly checked to be linearly independent. We can then claim it is a basis because it agrees with the expected number of structures obtained with a group theoretic formula introduced in [25] as a superspace generalization of [26].

As already mentioned, $t$ can be written as a linear combination

$$
t\left(Z ; \eta_{1}, \eta_{2}, \bar{\eta}_{2}, \eta_{3}\right)=\frac{1}{U^{3}} \sum_{k=1}^{10} \mathcal{C}_{k} \mathbb{T}_{k}\left(Z ; \eta_{1}, \eta_{2}, \bar{\eta}_{2}, \eta_{3}\right) .
$$

The explicit expressions for the $\mathbb{T}_{k}$ 's are

$$
\begin{aligned}
\mathbb{T}_{1} & =i[2 \overline{2}][13]^{j} & \mathbb{T}_{6} & =[12][1 \overline{2}][\Theta 3][3 \bar{\Theta}][13]^{j-2} \\
\mathbb{T}_{2} & =i[12][3 \overline{2}][13]^{j-1} & \mathbb{T}_{7} & =[12][\bar{\Theta} \overline{2}][\Theta 3][13]^{j-1} \\
\mathbb{T}_{3} & =[3 \bar{\Theta}][\Theta 2][1 \overline{2}][13]^{j-1} & \mathbb{T}_{8} & =[12][3 \overline{2}][\Theta \bar{\Theta}][13]^{j-1} \\
\mathbb{T}_{4} & =[\Theta 2][\bar{\Theta} \overline{2}][13]^{j} & \mathbb{T}_{9} & =i\left[\Theta^{2}\right]\left[\bar{\Theta}^{2}\right][2 \overline{2}][13]^{j} \\
\mathbb{T}_{5} & =[2 \overline{2}][\Theta \bar{\Theta}][13]^{j} & \mathbb{T}_{10} & =i\left[\Theta^{2}\right]\left[\bar{\Theta}^{2}\right][12][3 \overline{2}][13]^{j-1} .
\end{aligned}
$$

The factors of $i$ are introduced for later convenience. If $j=1$ then $\mathbb{T}_{6}$ is not present and if $j=0$ then $\mathbb{T}_{2,3,6,7,8,10}$ are not present. 


\section{Constraints on the supersymmetric three-point correlator}

\subsection{Conservation}

The superconformal multiplet $\mathcal{J}(\mathbf{z})$ contains the R-symmetry current, the supersymmetry current and the stress-energy tensor. As a consequence, it satisfies a shortening condition (see e.g. [27]) which can be expressed in superspace as

$$
D^{\alpha} \mathcal{J}_{\alpha \dot{\alpha}}(\mathbf{z})=\bar{D}^{\dot{\alpha}} \mathcal{J}_{\alpha \dot{\alpha}}(\mathbf{z})=0,
$$

with $D$ and $\bar{D}$ the superspace derivatives. In this subsection we will explore the consequences of this constraint on the correlator at separated points. In section 3.3 we will study the contact terms instead. At separated points the prefactor in (2.3) commutes with the conservation differential operators acting on $\mathbf{z}_{2},{ }^{9}$ thus we can express the conservation condition as an equation involving only $t$ and the variable $Z$ :

$$
\partial_{\eta_{2}} \mathcal{D} t\left(Z ; \eta_{i}, \bar{\eta}_{i}\right)=\partial_{\bar{\eta}_{2}} \overline{\mathcal{D}} t\left(Z ; \eta_{i}, \bar{\eta}_{i}\right)=0,
$$

where

$$
\mathcal{D}_{\alpha}=\frac{\partial}{\partial \Theta^{\alpha}}-i \sigma_{\alpha \dot{\alpha}}^{\mu} \bar{\Theta}^{\dot{\alpha}} \frac{\partial}{\partial U^{\mu}}, \quad \overline{\mathcal{D}}_{\dot{\alpha}}=\frac{\partial}{\partial \bar{\Theta}^{\dot{\alpha}}}+i \Theta^{\alpha} \sigma_{\alpha \dot{\alpha}}^{\mu} \frac{\partial}{\partial U^{\mu}} .
$$

Equation (3.2) imposes the following linear constraints for general $j>1$ :

$$
\mathcal{C}_{5}=-\mathcal{C}_{3}-2 \mathcal{C}_{4}, \quad \mathcal{C}_{7}=2 \mathcal{C}_{2}-\mathcal{C}_{3}-\mathcal{C}_{6}, \quad \mathcal{C}_{8}=-4 \mathcal{C}_{2}+2 \mathcal{C}_{3}+\mathcal{C}_{6}, \quad \mathcal{C}_{9}=\mathcal{C}_{10}=0 .
$$

When $j=1$ it suffices to set $\mathcal{C}_{6}$ to zero and when $j=0$ one simply has

$$
\mathcal{C}_{5}=-2 \mathcal{C}_{4}, \quad \mathcal{C}_{9}=0 .
$$

\subsection{Reality}

Since $\mathcal{O}$ and $\overline{\mathcal{O}}=\mathcal{O}^{\dagger}$ are conjugated to each other and $\mathcal{J}$ is hermitian, the correlator under study must be real. Concretely, we want to impose that

$$
\left\langle\overline{\mathcal{O}}\left(\mathbf{z}_{1}\right) \mathcal{J}\left(\mathbf{z}_{2}\right) \mathcal{O}\left(\mathbf{z}_{3}\right)\right\rangle^{*}=\left\langle\overline{\mathcal{O}}\left(\mathbf{z}_{3}\right) \mathcal{J}\left(\mathbf{z}_{2}\right) \mathcal{O}\left(\mathbf{z}_{1}\right)\right\rangle
$$

namely that taking the complex conjugation is the same as swapping points 1 and 3 . The prefactor in (2.3) is not invariant under this transformation, moreover the exchange $1 \leftrightarrow 3$ does not act nicely on $Z_{3}$. This means that we cannot translate the reality condition into a constraint for $t$ right away. ${ }^{10}$ We proceed, then, to expand the definition of (3.6)

$$
\begin{aligned}
\frac{\left(-\eta_{1} \mathrm{x}_{1 \overline{3}} \partial_{\bar{\chi}_{1}}\right)^{j} \eta_{2} \mathrm{x}_{2 \overline{3}} \partial_{\bar{\chi}_{2}} \partial_{\chi_{2}} \mathrm{x}_{3 \overline{2}} \bar{\eta}_{2}}{x_{\overline{3} 1}{ }^{2 q+j} x_{\overline{1} 3}{ }^{2 \bar{q}} x_{\overline{2} 3}{ }^{4} x_{\overline{3} 2}{ }^{4}} t\left(Z_{3} ; \chi_{1}, \chi_{2}, \bar{\chi}_{2}, \eta_{3}\right)^{*} & \\
= & \frac{\left(\partial_{\chi_{1}} \mathrm{x}_{1 \overline{3}} \bar{\eta}_{3}\right)^{j} \eta_{2} \mathrm{x}_{2 \overline{1}} \partial_{\bar{\chi} 2} \partial_{\chi_{2} \mathrm{x}_{1 \overline{2}} \bar{\eta}_{2}}}{x_{\overline{3} 1}{ }^{2 q+j} x_{\overline{1}}{ }^{2 \bar{q}} x_{\overline{1} 2}{ }^{4} x_{\overline{2} 1}{ }^{4}} t\left(\bar{Z}_{1} ; \chi_{1}, \chi_{2}, \bar{\chi}_{2}, \eta_{1}\right)
\end{aligned}
$$

\footnotetext{
${ }^{9}$ Due to $D_{2}^{\alpha}\left(\mathrm{x}_{2 \overline{3}}\right)_{\alpha \dot{\alpha}} / x_{\overline{3} 2}^{4}=\bar{D}_{2}^{\dot{\alpha}}\left(\mathrm{x}_{3 \overline{2}}\right)_{\alpha \dot{\alpha}} / x_{\overline{2} 3}^{4}=0$ when $x_{23} \neq 0$.

${ }^{10}$ This is obviously a consequence of our parametrization. In the ordering $\langle\mathcal{O} \overline{\mathcal{O}} \mathcal{J}\rangle$ the reality condition can be solved easily. On the other hand we would lose the fact that the conservation operator commutes with the prefactor thus making conservation much harder to impose.
} 
where we defined, $\bar{Z}_{1}=\left(-\bar{X}_{1},-\Theta_{1},-\bar{\Theta}_{1}\right)$. The prefactor appearing in the above expression can be recast in terms of the supersymmetric inversion tensor $I^{i \bar{\imath}}$ introduced in [18], which we review in appendix A. The indices $i$ (resp. $\bar{l}$ ) collectively denote $j$ symmetrized $\alpha$ (resp. $(\dot{\alpha}))$ indices and $\mu$ is an ordinary Lorentz vector index. In this notation (3.7) reads

$$
\frac{x_{\overline{2} 1}{ }^{3} x_{\overline{1} 2}{ }^{3}}{x_{\overline{2}}{ }^{3} x_{\overline{3} 2}{ }^{3}} I^{i_{1} \bar{\imath}_{1}}\left(x_{1 \overline{3}}\right) I_{\mu \nu}\left(x_{2 \overline{3}}, x_{\overline{2} 3}\right)\left(t^{*}\right)_{\bar{\imath}_{1}}{ }^{\nu} \bar{\imath}_{3}\left(Z_{3}\right)=\bar{I}_{\bar{l}_{3} i_{3}}\left(x_{\overline{3} 1}\right) I_{\mu \nu}\left(x_{2 \overline{1}}, x_{\overline{2} 1}\right) t^{i_{3} \nu i_{1}}\left(\bar{Z}_{1}\right) .
$$

Contracting both sides of this expression with $\bar{I}^{\sigma \lambda}\left(\bar{X}_{1}, X_{1}\right) I^{i_{4} \bar{\imath}_{3}}\left(x_{1 \overline{3}}\right) I_{\lambda \rho}\left(x_{1 \overline{3}}, x_{\overline{1} 3}\right)$ $\bar{I}^{\rho \mu}\left(x_{\overline{3} 2}, x_{3 \overline{2}}\right)$ and using the various identities listed in appendix $\mathrm{A}$ we get to the final expression

$$
I^{i_{1} \bar{\imath}_{1}}\left(\bar{X}_{1}\right) I^{i_{4} \bar{\imath}_{3}}\left(\bar{X}_{1}\right)\left(t^{*}\right)_{\bar{\imath}_{1}}{ }^{\sigma} \bar{\imath}_{3}\left(Z_{1}\right)=t^{i_{4} \sigma i_{1}}\left(\bar{Z}_{1}\right)
$$

which, in index-free form, reads ${ }^{11}$

$$
(-1)^{j} \bar{X}^{-2 j}\left(t^{*}\right)\left(Z ; \overline{\mathrm{X}} \eta_{1}, \eta_{2}, \bar{\eta}_{2}, \overline{\mathrm{X}} \eta_{3}\right)=t\left(\bar{Z} ; \eta_{3}, \eta_{2}, \bar{\eta}_{2}, \eta_{1}\right)
$$

We can then solve this equation much more easily. In doing so we find the following linear constraints for even $j>1$ :

$$
\begin{aligned}
\mathcal{C}_{1}{ }^{*} & =\mathcal{C}_{1}, & \mathcal{C}_{2}{ }^{*} & =\mathcal{C}_{2}, \\
\mathcal{C}_{3}{ }^{*} & =2 \mathcal{C}_{2}-\mathcal{C}_{6}-\mathcal{C}_{7}, & \mathcal{C}_{4}{ }^{*} & =-2 \mathcal{C}_{2}+\mathcal{C}_{3}+\mathcal{C}_{4}+\mathcal{C}_{6}+\mathcal{C}_{7} \\
\mathcal{C}_{5}{ }^{*} & =\mathcal{C}_{5}, & \mathcal{C}_{6}{ }^{*} & =\mathcal{C}_{6} \\
\mathcal{C}_{7}{ }^{*} & =2 \mathcal{C}_{2}-\mathcal{C}_{3}-\mathcal{C}_{6}, & \mathcal{C}_{8}{ }^{*} & =\mathcal{C}_{8} \\
\mathcal{C}_{9}{ }^{*} & =\mathcal{C}_{2}-\frac{1}{2}\left(\mathcal{C}_{3}+\mathcal{C}_{6}+\mathcal{C}_{7}\right)+\mathcal{C}_{9}, & \mathcal{C}_{10}{ }^{*} & =-2 \mathcal{C}_{2}+\mathcal{C}_{3}+\mathcal{C}_{6}+\mathcal{C}_{7}+\mathcal{C}_{10}
\end{aligned}
$$

If $j$ is odd the equations are obtained by adding an overall minus sign on the right hand side. If $j=1$ it is sufficient to set $\mathcal{C}_{6}=\mathcal{C}_{6}{ }^{*}=0$. For $j=0$ instead one has simply

$$
\mathcal{C}_{1}{ }^{*}=\mathcal{C}_{1}, \quad \mathcal{C}_{4}{ }^{*}=\mathcal{C}_{4}, \quad \mathcal{C}_{5}{ }^{*}=\mathcal{C}_{5}, \quad \mathcal{C}_{9}{ }^{*}=\mathcal{C}_{9}
$$

Combined with conservation (3.4), these equations imply that the remaining $\mathcal{C}_{k}$ are purely real (resp. imaginary) if $j$ is even (resp. odd).

\subsection{Ward identities}

There are in principle two ways to impose the Ward identities: one could apply them directly in superspace with the formalism of [18], or alternatively one could expand the correlator in components and apply the nonsupersymmetric Ward identity to each triplet of superdescendants. Since we already need the three-point function in components to make contact with the ANEC and since nonsupersymmetric Ward identities are much easier to compute, we opted for the second approach. We did not explore all possible combinations of superdescendants but we observed that after imposing the identities for $\langle\bar{O} J O\rangle$ and

\footnotetext{
${ }^{11} \mathrm{By}\left(t^{*}\right)(Z ; \ldots)$ we mean: first apply the complex conjugation to $t\left(Z ; \eta_{1}, \eta_{2}, \bar{\eta}_{2}, \eta_{3}\right)$, then replace $\bar{\eta}_{1(3)}$ with $\overline{\mathrm{X}} \eta_{1(3)}$.
} 
$\langle\bar{O} T O\rangle$, all other choices of superdescendants that we investigated were not yielding any new constraints. By $O, \bar{O}$ we mean the lowest component of $\mathcal{O}, \overline{\mathcal{O}}$, while the R-current $J_{\mu}$ and the stress-energy tensor $T_{\mu \nu}$ are, respectively, the lowest component and the $Q \bar{Q}$ component of $\mathcal{J}$. We will also denote the supersymmetry currents as $S_{\alpha}^{\mu}$ and $\bar{S}_{\dot{\alpha}}^{\mu}$. They are, respectively, the $Q$ and the $\bar{Q}$ components of $\mathcal{J}$.

For nonsupersymmetric three-point functions we use the conventions of [28]. ${ }^{12}$ A threepoint function $t$ can be expressed as a prefactor multiplying a linear combination of tensor structures,

$$
\mathrm{t}_{O_{1} O_{2} O_{3}}\left(x_{1,2,3}, \eta_{1,2,3}, \bar{\eta}_{1,2,3}\right) \equiv\left\langle\prod_{i=1}^{3} O_{i}\left(x_{i}, \eta_{i}, \bar{\eta}_{i}\right)\right\rangle=\mathcal{K} \sum_{k} \lambda_{k} \mathcal{T}_{k}\left(x_{1,2,3}, \eta_{1,2,3}, \bar{\eta}_{1,2,3}\right),
$$

where $\mathcal{K}$ is of the form $\mathcal{K}=\prod_{j>i} x_{i j}{ }^{\delta_{i j}}, \delta_{i j}$ being linear functions of the dimensions and spins of the operators in the three-point function. The tensor structures $\mathcal{T}_{k}$ can be built out of the following invariants:

$$
\mathbb{I}^{i j}, \quad \mathbb{J}_{i j}^{k}, \quad \mathbb{K}_{k}^{i j}, \quad \overline{\mathbb{K}}_{k}^{i j} .
$$

We refer the reader to [28, appendix D] for their definition. For the two-point function we use the convention

$$
\mathrm{n}_{\bar{O} O}\left(x_{12}, \eta_{1,2}, \bar{\eta}_{1,2}\right) \equiv\left\langle\bar{O}\left(x_{1}, \eta_{1}, \bar{\eta}_{1}\right) O\left(x_{2}, \eta_{2}, \bar{\eta}_{2}\right)\right\rangle=c_{O} i^{j+\bar{\jmath}} \frac{\left(\eta_{2} \mathrm{x}_{12} \bar{\eta}_{1}\right)^{j}\left(\eta_{1} \mathrm{x}_{12} \bar{\eta}_{2}\right)^{\bar{\jmath}}}{x_{12}{ }^{2 \Delta+j+\bar{\jmath}}},
$$

assuming $O$ has spin $\left(\frac{1}{2} j, \frac{1}{2} \bar{\jmath}\right)$. In a unitary theory $c_{O}>0$. The coefficient $c_{O}$ is usually set to 1 by normalizing the operator in the two-point function, but here we do not do this rescaling of operator because in the supersymmetric case the relative normalizations of the operators in the same superconformal multiplet are fixed. We will assume that the superconformal primary is normalized to $c_{O}=1$ and use the results of [29] to fix the normalization of its superdescendants.

R-current. Let us start our analysis with the Ward identity for the $\mathrm{U}(1)_{R}$ symmetry. The three-point function under study is $\mathrm{t}_{\bar{O} J O}$, where $O$ is any operator with charge $r$ under $\mathrm{U}(1)_{R}$ and $\bar{O}$ its conjugate (with charge $-r$ ). Now consider a codimension-one surface $\Sigma$ enclosing $x_{2}$ and $x_{3}$ but not $x_{1}$. The Ward identity states

$$
\frac{i}{2} \int_{\Sigma} \mathrm{d} \Omega\left(x_{23}\right) x_{23}^{2} \partial_{\eta_{2}} \mathrm{x}_{23} \partial_{\bar{\eta}_{2}} \mathrm{t}_{\bar{O} J O}\left(x_{i} ; \eta_{i}, \bar{\eta}_{i}\right)=\mathfrak{N} r \mathrm{n}_{\bar{O} O}\left(x_{13}, \eta_{1,3}, \bar{\eta}_{1,3}\right) .
$$

The factor $i / 2$ on the left hand side comes from the $-1 / 2$ obtained from $x^{\mu} J_{\mu}=-\frac{1}{2} \tilde{\mathrm{x}}^{\dot{\alpha} \alpha} J_{\alpha \dot{\alpha}}$ and $\mathrm{a}-i$ from the Wick rotation. Indeed the integral in the above equation is in Euclidean signature and the right prescription for the Wick rotation is the one that keeps the operators radially ordered as indicated, namely if $x_{i}^{0}=-i \tau_{i}$, then $\tau_{1}>\tau_{2}>\tau_{3}$. The constant $\mathfrak{N}$ is a normalization for the multiplet $\mathcal{J}$. In order to match the conventions of [10] we must set

\footnotetext{
${ }^{12}$ We used their Mathematica package CFTs4D to generate the tensor structures.
} 
$\mathfrak{N}=2$. Since this integral depends only topologically on the points we can evaluate it in the simplified limit $x_{1} \rightarrow \infty, x_{23} \rightarrow 0$. The results for an operator $O$ of spin $\left(\frac{1}{2} j, 0\right)$ are summarized in table 3 and those for $O$ of spin $\left(\frac{1}{2} j, \frac{1}{2}\right)$ in table $4 .{ }^{13}$

Stress-energy tensor. We proceed by considering the Ward identities for the conformal group. To each conformal Killing vector $\varepsilon_{\mu}^{a}$ is associated a possibly independent identity. It is sufficient to impose only $\varepsilon_{\mu}=x_{\mu}$ (dilatations) and $\varepsilon_{\mu}^{\nu}=\delta_{\mu}^{\nu}$ (translations). ${ }^{14}$ Dilatations and translations imply respectively the identities

$$
\begin{gathered}
-\frac{i}{8} \int_{\Sigma} \mathrm{d} \Omega\left(x_{23}\right) x_{23}^{2} \partial_{\eta_{2}} \mathrm{x}_{23} \partial_{\bar{\eta}_{2}} \partial_{\eta_{2}} \mathrm{x}_{2} \partial_{\bar{\eta}_{2}} \mathrm{t}_{\bar{O} T O}\left(x_{i}, \eta_{i}, \bar{\eta}_{i}\right) \\
=-i \mathfrak{N}\left(\Delta+x_{3} \cdot \partial_{3}\right) \mathrm{n}_{\bar{O} O}\left(x_{13}, \eta_{1,3}, \bar{\eta}_{1,3}\right) \\
-\frac{i}{8} \int_{\Sigma} \mathrm{d} \Omega\left(x_{23}\right) x_{23}{ }^{2} \partial_{\eta_{2}} \mathrm{x}_{23} \partial_{\bar{\eta}_{2}} \partial_{\eta_{2}} \mathrm{y}_{\bar{\eta}_{2}} \mathrm{t}_{\bar{O} T O}\left(x_{i}, \eta_{i}, \bar{\eta}_{i}\right) \\
=-i \mathfrak{N} y \cdot \partial_{3} \mathrm{n}_{\bar{O} O}\left(x_{13}, \eta_{1,3}, \bar{\eta}_{1,3}\right) \\
=
\end{gathered}
$$

where $y^{\mu}$ is an arbitrary vector used to contract the free index of the translation Killing vector. The operator $O$ in the above expression can be regarded to be the superconformal primary of spin $\left(\frac{1}{2} j, 0\right)$, in which case the result is summarized in table 5 . We can also replace $O \rightarrow \bar{Q} O$ of spin $\left(\frac{1}{2} j, \frac{1}{2}\right)$ whose results are in table 6 . Finally one could also consider $O \rightarrow Q O$; the result is obtained by a simple rescaling of the coefficients in table 5 and a replacement $j \rightarrow j \pm 1$. For the reader's convenience we report here the relative normalizations for the operators in the $\mathcal{O}$ multiplet as derived in [29]:

$$
\frac{c_{(Q O)^{(j+1,0)}}}{c_{O}}=2 \frac{j+2 q}{(j+1)^{2}}, \quad \frac{c_{(Q O)^{(j-1,0)}}}{c_{O}}=2 \frac{(j+1)(2 q-j-2)}{j}, \quad \frac{c_{(\bar{Q} O)}}{c_{O}}=4 \bar{q} .
$$

Supersymmetry current. For this Ward identity let us fix the third operator to be $O$. We then have three choices: $\mathrm{t}_{(Q \bar{O}) \bar{S} O}$ and $\mathrm{t}_{\left(\bar{Q} \bar{O}^{ \pm}\right) S O}$, where we used $\bar{Q} \bar{O}^{ \pm}$as a shorthand for $(\bar{Q} \bar{O})^{(j \pm 1,0)}$. The topological operator obtained by integrating $S$ or $\bar{S}$ over $\Sigma$ is precisely the supercharge $Q$ or $\bar{Q}$ respectively. We thus readily obtain the following identities

$$
\begin{gathered}
\frac{i}{2} \int_{\Sigma} \mathrm{d} \Omega\left(x_{23}\right) x_{23}^{2} \partial_{\eta_{2}} \mathrm{x}_{23} \partial_{\bar{\eta}_{2}} \mathrm{t}_{(Q \bar{O}) \bar{S} O}\left(x_{i} ; \eta_{i}, \bar{\eta}_{i}\right) \\
=\mathfrak{N} \bar{\eta}_{2} \partial_{\bar{\eta}_{3}} \mathrm{n}_{(Q \bar{O})(\bar{Q} O)}\left(x_{13}, \eta_{1,3}, \bar{\eta}_{1,3}\right), \\
\frac{i}{2} \int_{\Sigma} \mathrm{d} \Omega\left(x_{23}\right) x_{23}^{2} \partial_{\eta_{2}} \mathrm{x}_{23} \partial_{\bar{\eta}_{2}} \mathrm{t}_{\left(\bar{Q} \bar{O}^{+}\right) S O}\left(x_{i} ; \eta_{i}, \bar{\eta}_{i}\right) \\
=\mathfrak{N} \eta_{2} \partial_{\eta_{3}} \mathrm{n}_{\left(\bar{Q} \bar{O}^{+}\right)\left(Q O^{+}\right)}\left(x_{13}, \eta_{1,3}, \bar{\eta}_{1,3}\right),
\end{gathered}
$$

\footnotetext{
${ }^{13}$ The results showed in these tables and the subsequent ones already assume the normalization $\mathfrak{N}=2$.

${ }^{14}$ Following [30, appendix B] the independent constraints given by the Ward identities are as many as the number of singlets in

$$
\rho_{O} \otimes \rho_{\bar{O}}^{\dagger} \otimes(\bullet \oplus(1,0) \oplus(0,1))
$$

$\rho_{O}$ representing the Lorentz representation of $O$ and $\bullet$ the singlet. For $\rho_{O}=\left(\frac{1}{2} j, 0\right)$ the tensor product contains two singlets (one if $j=0$ ) and for $\rho=\left(\frac{1}{2} j, \frac{1}{2}\right)$ it contains three singlets (two if $j=0$ ). The equations (3.16) yield the exact same number of independent constraints.
} 


$$
\begin{aligned}
\frac{i}{2} \int_{\Sigma} \mathrm{d} \Omega\left(x_{23}\right) x_{23}{ }^{2} \partial_{\eta_{2}} \mathrm{x}_{23} \partial_{\bar{\eta}_{2}} \mathrm{t}_{\left(\bar{Q} \bar{O}^{-}\right) S O}\left(x_{i} ; \eta_{i}, \bar{\eta}_{i}\right) \\
=\mathfrak{N} \frac{j}{j+1} \eta_{2} \eta_{3} \mathrm{n}_{\left(\bar{Q} \bar{O}^{-}\right)\left(Q O^{-}\right)}\left(x_{13}, \eta_{1,3}, \bar{\eta}_{1,3}\right)
\end{aligned}
$$

The two-point functions must be normalized according to eq. (3.17). All the results are summarized in tables 7,8 and 9 .

\subsection{Shortening conditions}

The possible shortening conditions on the superconformal multiplet $\mathcal{O}$ have been classified in [19]. In this section we will explore all of them. On the algebra generated by $Q$ we can have the shortening conditions

$L$ : Unconstrained action on $\mathcal{O}$ (no null states). Unitarity bound $q \geqslant \frac{1}{2} j+1$.

$A_{1}$ : Null state $(Q O)^{(j-1, \bar{\jmath})}, j \geqslant 1$. Unitarity bound $q=\frac{1}{2} j+1$.

$A_{2}$ : Null state $\left(Q^{2} O\right)^{(0, \bar{\jmath})}, j=0$. Unitarity bound $q=1$.

$B:$ Null state $(Q O)^{(1, \bar{\jmath})}, j=0$. Unitarity bound $q=0$.

The same applies to the algebra generated by $\bar{Q}$. Therefore, a shortening condition on a superconformal multiplet can be described by specifying a choice of $\mathcal{X}_{i}=L, A_{1}, A_{2}, B$ for each of the two subalgebras: $\left[\mathcal{X}_{L}, \overline{\mathcal{X}}_{R}\right]$. For simplicity we will refer to $[L, \bar{B}]$ as chirality. Furthermore the conditions $\left[\mathcal{X}_{L}, \bar{A}_{1}\right]$ are absent because we are considering the case $\bar{\jmath}=0$. Since $\overline{\mathcal{O}}=\mathcal{O}^{\dagger}, \overline{\mathcal{O}}$ will satisfy the conjugate shortening $\left[\mathcal{X}_{R}, \overline{\mathcal{X}}_{L}\right]$. However, after imposing reality, either one of the two conditions is sufficient.

Shortening $\boldsymbol{B}$ or $\bar{B}$. For the $\bar{Q} O=0$ case $(\bar{q}=0)$ the prefactor of $(2.3)$ does not depend on $x_{\overline{3} 1}$, while for the $Q O=0$ case $(q=j=0)$ the prefactor does not depend on $x_{\overline{1} 3}$. In both cases we can commute the superspace derivative and obtain conditions on $t$ only. They read, respectively,

$$
\bar{\eta}_{1} \overline{\mathcal{D}} t\left(Z ; \eta_{i}, \bar{\eta}_{i}\right)=0, \quad \eta_{1} \mathcal{D} t\left(Z ; \eta_{i}, \bar{\eta}_{i}\right)=0
$$

Shortening $\boldsymbol{A}_{\mathbf{1}}$. Also in this case (when $q=j / 2+1$ ) we can commute the differential operator with the prefactor due to

$$
\partial_{\bar{\eta}_{1}} \bar{D}_{1} \frac{\left(\eta_{1} \mathrm{x}_{3 \overline{1}} \bar{\eta}_{1}\right)^{j}}{x_{\overline{1} 3}{ }^{2 j+2}}=0, \quad \text { for } x_{13} \neq 0
$$

and thus we readily obtain

$$
\partial_{\eta_{1}} \mathcal{D} t\left(Z ; \eta_{i}, \bar{\eta}_{i}\right)=0
$$




$$
\text { (a) } j>1
$$

\begin{tabular}{|c|c|c|c|}
\hline & $\bar{L}$ & $\bar{A}_{2}$ & $\bar{B}$ \\
\hline$L$ & 2 & 2 & 0 \\
\hline$A_{1}$ & 1 & 1 & I \\
\hline
\end{tabular}

(b) $j=1$

\begin{tabular}{|c|c|c|c|}
\hline & $\bar{L}$ & $\bar{A}_{2}$ & $\bar{B}$ \\
\hline$L$ & 1 & 1 & 0 \\
\hline$A_{1}$ & 1 & 1 & 0 \\
\hline
\end{tabular}

(c) $j=0$

\begin{tabular}{l|lll|}
\multicolumn{1}{l|}{} & $\bar{L}$ & $\bar{A}_{2}$ & $\bar{B}$ \\
\cline { 2 - 4 }$L$ & 0 & 0 & 0 \\
$A_{2}$ & 0 & 0 & 0 \\
$B$ & 0 & 0 & $\mathbf{0}$ \\
\cline { 2 - 4 } & & &
\end{tabular}

Table 1. Number of independent coefficients $\mathcal{C}_{k}$ of the superspace correlator as different shortening conditions are chosen. The slash means that there is no consistent three-point function. The boldface zero means that the three-point function is identically zero. Other zeros imply that the three-point function is completely fixed in terms of $q, \bar{q}$ and $j$. In all cases these numbers refer to real degrees of freedom as the $\mathcal{C}_{k}$ are either all real or all purely imaginary.

Shortening $\boldsymbol{A}_{2}$ or $\overline{\boldsymbol{A}}_{2}$. In this case the commutation of the derivative and the prefactor is due to the identities

$$
\bar{D}_{1}^{2} \frac{1}{x_{\overline{1} 3}^{2}}=D_{1}^{2} \frac{1}{x_{\overline{3} 1}^{2}}=0, \quad \text { for } x_{13} \neq 0 .
$$

Thus for $Q^{2} O=0(q=1$ and $j=0)$ and for $\bar{Q}^{2} O=0(\bar{q}=1)$ we get, respectively

$$
\mathcal{D}^{2} t\left(Z ; \eta_{i}, \bar{\eta}_{i}\right)=0, \quad \overline{\mathcal{D}}^{2} t\left(Z ; \eta_{i}, \bar{\eta}_{i}\right)=0 .
$$

In table 2 we summarize all the constraints arising from (3.19), (3.21) and (3.23). All shortening conditions can be easily obtained by combining them. table 1 instead shows how many independent coefficients are left in the superspace correlator as we choose different shortening conditions and impose all other constraints obtained before.

\section{Expansion of the superspace correlator}

In order to apply the various constraints originating from the ANEC to our three-point function in superspace we need to express its components in a basis of nonsupersymmetric three-point functions. This will be achieved by Taylor expanding in the Grassmann coordinates $\theta_{i}, \bar{\theta}_{i}$. We relied on a Mathematica package ${ }^{15}$ to perform the spinor algebra involved in this computation. Due to the Schouten identities mentioned above, it is hard to determine whether two quantities are equal. Therefore we check for equality by replacing the various quantities that appear with random numerical values. ${ }^{16}$

Every order that contains at least a $\theta$ and a $\bar{\theta}$ at the same point will mix with conformal descendants due to $\left\{Q_{\alpha}, \bar{Q}_{\dot{\alpha}}\right\}=2 \mathrm{P}_{\alpha \dot{\alpha}}$. The results of [29] can be used to subtract these contributions. We will only perform this expansion to first order in $\theta_{i}, \bar{\theta}_{i}$ and not for all possible combinations but only the ones of interest. We also performed the expansion to

\footnotetext{
${ }^{15}$ Which can be made available upon request.

${ }^{16}$ After sufficiently many replacements, this is equivalent to picking a basis at random and checking for equality for every vector in it. The fact that we replace numerical values to Grassmann coordinates is not an issue if one orders the factors in a canonical way before applying the replacement. Moreover there are no precision issues because we use exact rational numbers.
} 


\begin{tabular}{|c|c|c|}
\hline & Constraints & Conditions \\
\hline$A_{1}$ & $\begin{array}{l}\mathcal{C}_{6}=(j-1) \mathcal{C}_{3}+\frac{j(j-1)}{j+1}\left(\mathcal{C}_{5}-4 \mathcal{C}_{1}\right), \\
\mathcal{C}_{7}=-2 \mathcal{C}_{2}+\mathcal{C}_{3}+j \mathcal{C}_{4}+\frac{2 j}{j+1}\left(\mathcal{C}_{5}+(j-3) \mathcal{C}_{1}\right), \\
\mathcal{C}_{8}=4 \mathcal{C}_{2}-\mathcal{C}_{3}+\frac{2 j}{1+j}\left(4 \mathcal{C}_{1}-\mathcal{C}_{5}\right), \\
\mathcal{C}_{10}=j \mathcal{C}_{9}=j \mathcal{C}_{4}+\frac{j}{2}\left(\mathcal{C}_{3}+\mathcal{C}_{5}\right) .\end{array}$ & $j \geqslant 1, q=j / 2+1$ \\
\hline$A_{2}$ & $\mathcal{C}_{9}=\mathcal{C}_{4}+\frac{1}{2} \mathcal{C}_{5}$ & $j=0, q=1$ \\
\hline $\bar{A}_{2}$ & $\begin{array}{l}\mathcal{C}_{9}=-\frac{1}{2}\left(\mathcal{C}_{3}+\mathcal{C}_{5}\right)-\mathcal{C}_{4} \\
\mathcal{C}_{10}=-\frac{1}{2}\left(\mathcal{C}_{6}+\mathcal{C}_{8}\right)-\mathcal{C}_{7}\end{array}$ & $\bar{q}=1$ \\
\hline$B$ & $\mathcal{C}_{4}=-2 \mathcal{C}_{1}, \quad \mathcal{C}_{5}=4 \mathcal{C}_{1}, \quad \mathcal{C}_{9}=0$ & $j=0, q=0$ \\
\hline $\bar{B}$ & $\begin{array}{l}\mathcal{C}_{4}=2 \mathcal{C}_{1}, \quad \mathcal{C}_{5}=-4 \mathcal{C}_{1}, \\
\mathcal{C}_{7}=2 \mathcal{C}_{2}, \quad \mathcal{C}_{8}=-4 \mathcal{C}_{2}, \\
\mathcal{C}_{3}=\mathcal{C}_{6}=\mathcal{C}_{9}=\mathcal{C}_{10}=0\end{array}$ & $\bar{q}=0$ \\
\hline
\end{tabular}

Table 2. Constraints on the coefficients $\mathcal{C}_{k}$ following from the various shortening conditions on the multiplet $\mathcal{O}$ (here $\bar{\jmath}=0$ is implicit). Case $A_{1}$ for $j=1$ and cases $\bar{A}_{2}$ and $\bar{B}$ for $j=0,1$ can be obtained by setting to zero the absent coefficients $\left(\mathcal{C}_{6}\right.$ for $j=1$ and $\mathcal{C}_{2,3,6,7,8,10}$ for $\left.j=0\right)$.

all orders in $\theta_{2}, \bar{\theta}_{2}$ and to all orders in $\theta_{1}, \bar{\theta}_{1}$ to make some consistency checks, ${ }^{17}$ but we will not present these results here. For nonsupersymmetric three-point functions we will remain consistent with the conventions introduced in section 3.3.

\subsection{Lowest order}

At this order we simply have $J$. Consistently with the previous sections we denote the three-point function coefficients by

$$
\mathrm{t}_{\bar{O} J O} \longrightarrow C_{k}
$$

The results, without assuming the reality condition and conservation, are shown in table 10 .

\subsection{Three-point function $\langle\bar{O} T O\rangle$}

At order $\theta_{2} \bar{\theta}_{2}$ we have the stress-energy tensor. Consistently with the previous sections we denote the three-point function coefficients by

$$
\mathrm{t}_{\bar{O} T O} \longrightarrow D_{k}
$$

\footnotetext{
${ }^{17}$ Namely we observed that the order $\theta_{2}^{2} \bar{\theta}_{2}^{2}$ consists only of descendants when the conservation condition (3.4) is applied, consistently with the operator content of $\mathcal{J}$. In addition we verified that applying the shortening differential operators in section 3.4 on the expanded correlator yields the same constraints.
} 
The results are shown in table 11 . The conservation of $\mathcal{J}$ and the reality condition are not assumed there. In principle the expansion also contains superdescendants of $\mathcal{J}$ of spin $(0,0),(0,1)$ and $(1,0)$. We checked that those contributions vanish after imposing conservation and we will not report those results here.

\subsection{Three-point functions $\langle(\bar{Q} \bar{O}) S O\rangle$ and $\langle(Q \bar{O}) \bar{S} O\rangle$}

At order $\theta_{1} \bar{\theta}_{2}, \bar{\theta}_{1} \theta_{2}$ we have the supersymmetry current with the first superdescendant of $\overline{\mathcal{O}}$. The naming of the coefficients is

$$
\mathrm{t}_{\left(\bar{Q} \bar{O}^{+}\right) S O} \longrightarrow E_{k}, \quad \mathrm{t}_{\left(\bar{Q} \bar{O}^{-}\right) S O} \longrightarrow F_{k}, \quad \mathrm{t}_{(Q \bar{O}) \bar{S} O} \longrightarrow G_{k} .
$$

As before $\bar{Q} \bar{O}^{ \pm}$stands for $(\bar{Q} \bar{O})^{(0, j \pm 1)}$. Also in these cases the results are presented without conservation and reality applied - they can be found in tables 12, 13 and 14 . There are also contributions from superdescendants of spin $\left(0, \frac{1}{2}\right)$ or $\left(\frac{1}{2}, 0\right)$. As in the previous subsection we have verified that they vanish after conservation is imposed and we will not report those results.

\subsection{Three-point functions $\langle(\bar{Q} \bar{O}) J(Q O)\rangle$ and $\langle(Q \bar{O}) J(\bar{Q} O)\rangle$}

At order $\theta_{1} \bar{\theta}_{3}, \bar{\theta}_{1} \theta_{3}$ we extract the descendants $Q O, \bar{Q} O$ and their conjugates. We need this mainly as a preliminary result for the computation of the next subsection. We named

$$
\begin{aligned}
\mathrm{t}_{\left(\bar{Q} \bar{O}^{+}\right) J\left(Q O^{+}\right)} & \longrightarrow N_{k}, \quad \mathrm{t}_{\left(\bar{Q} \bar{O}^{+}\right) J\left(Q O^{-}\right)} \longrightarrow O_{k}, \\
\mathrm{t}_{\left(\bar{Q} \bar{O}^{-}\right) J\left(Q O^{+}\right)} & \longrightarrow P_{k}, \quad \mathrm{t}_{\left(\bar{Q} \bar{O}^{-}\right) J\left(Q O^{-}\right)} \longrightarrow Q_{k},
\end{aligned}
$$

where $\left(Q O^{ \pm}\right)$stands for $(Q O)^{(j \pm 1,0)}$, and

$$
\mathrm{t}_{(Q \bar{O}) J(\bar{Q} O)} \longrightarrow I_{k}
$$

In order to make the computation more manageable, this time we applied conservation and reality from the start. The results are in tables 15, 16, 17, 18 and 19.

\subsection{Three-point functions $\langle(\bar{Q} \bar{O}) T(Q O)\rangle$ and $\langle(Q \bar{O}) T(\bar{Q} O)\rangle$}

At order $\theta_{1} \theta_{2} \bar{\theta}_{2} \bar{\theta}_{3}, \bar{\theta}_{1} \theta_{2} \bar{\theta}_{2} \theta_{3}$ we extract the descendants $Q O, \bar{Q} O$ and their conjugates coupled with the stress tensor. These terms are needed in order to impose the ANEC on superconformal descendants inside $\mathcal{O}$. We named

$$
\begin{aligned}
\mathrm{t}_{\left(\bar{Q} \bar{O}^{+}\right) T\left(Q O^{+}\right)} & \longrightarrow J_{k}, \\
\mathrm{t}_{\left(\bar{Q} \bar{O}^{-}\right) T\left(Q O^{+}\right)} & \longrightarrow L_{k}, \\
\mathrm{t}_{(Q \bar{Q}) T(\bar{Q} O)} & \longrightarrow H_{k} .
\end{aligned}
$$

Also this time we applied conservation and reality from the start. The results are in tables 20, 21, 22, 23 and 24 . 


\section{The averaged null energy condition}

Following $[10,31]$ we define the state $|\psi\rangle$ of (1.1) by acting with some operator $O(x, \eta, \bar{\eta})$ on the CFT vacuum $|0\rangle$. Then we take the Fourier transform in order to give the state a definite momentum, ${ }^{18}$ which can be set to $q^{\mu}=(1, \mathbf{0})$ without loss of generality. Next we multiply by $\left(x^{+}\right)^{2} / 16$ and send $x^{+} \rightarrow \infty$ to simplify the computations. Lastly we need to specify a polarization, but using the auxiliary spinors $\eta$ and $\bar{\eta}$ we can obtain all possible polarizations at once.

The ANEC integral breaks rotation invariance to an $\mathrm{SO}(2)$ generated by $\sigma^{12}{ }_{\alpha}{ }^{\beta}$ and $\bar{\sigma}^{12^{\dot{\alpha}}}{ }_{\dot{\beta}}$ in the respective representations. Under a $\varphi$ rotation of this subgroup, fundamental spinors with a lower index transform as follows:

$$
\left(\begin{array}{l}
a \\
b
\end{array}\right)_{\alpha} \longrightarrow\left(\begin{array}{l}
a e^{-i \varphi / 2} \\
b e^{i \varphi / 2}
\end{array}\right)_{\alpha}, \quad\left(\begin{array}{l}
\bar{a} \\
\bar{b}
\end{array}\right)_{\dot{\alpha}} \longrightarrow\left(\begin{array}{l}
\bar{a} e^{i \varphi / 2} \\
\bar{b} e^{-i \varphi / 2}
\end{array}\right)_{\dot{\alpha}} .
$$

This will help us in the following way: in principle, if there are $s$ choices for the polarization of $O$ and $\bar{O}$ one would have to apply the ANEC integral to each pair of choices, diagonalize an $s \times s$ matrix and require the positivity of each eigenvalue (or equivalently require semidefinite positiveness of an $s \times s$ matrix). This rotational symmetry reduces the matrix to a block diagonal form, making much simpler the study of its positiveness.

\subsection{Operators of $\operatorname{spin}\left(\frac{1}{2} j, 0\right)$}

Let us focus first on the case where $O(x, \eta, \bar{\eta})$ has spin $\left(\frac{1}{2} j, 0\right)$. We can expand the $\eta$ 's in the eigenbasis of the $\mathrm{SO}(2)$ spin,

$$
\eta_{3}^{\alpha}=\left(\begin{array}{c}
m \\
p
\end{array}\right) \equiv m \boldsymbol{\xi}_{-}^{\alpha}+p \boldsymbol{\xi}_{+}^{\alpha}, \quad \bar{\eta}_{1}^{\dot{\alpha}}=\left(\begin{array}{c}
\bar{p} \\
\bar{m}
\end{array}\right) \equiv \bar{p} \overline{\boldsymbol{\xi}}_{+}^{\dot{\alpha}}+\bar{m} \overline{\boldsymbol{\xi}}_{-}^{\dot{\alpha}},
$$

where the redundancy $\boldsymbol{\xi}_{ \pm}=\overline{\boldsymbol{\xi}}_{\mp}$ has been introduced for convenience. The stress tensor is instead polarized along the null geodesic $u^{\mu}$, which is translated to

$$
\eta_{2}^{\alpha}=\boldsymbol{\xi}_{-}^{\alpha}, \quad \bar{\eta}_{2}^{\dot{\alpha}}=\overline{\boldsymbol{\xi}}_{+}^{\dot{\alpha}} .
$$

Now we can perform the ANEC integral (1.1) with the prescriptions defined above on an arbitrary three-point function $\mathrm{t}_{\bar{O} T O} \cdot{ }^{19}$ We define $x_{13}=x, x_{23}=y$ and

$$
\left.\mathcal{A}\left[\mathrm{t}_{\bar{O} T O}\right] \equiv \int_{-\infty}^{\infty} \mathrm{d} y^{-} \lim _{y^{+} \rightarrow \infty} \frac{\left(y^{+}\right)^{2}}{16} \int_{\mathbb{R}^{4}} \mathrm{~d}^{4} x e^{-i x^{0}} \mathrm{t}_{\bar{O} T O}\left(x, y ; \bar{\eta}_{1}, \eta_{2}, \bar{\eta}_{2}, \eta_{3}\right)\right|_{\substack{\bar{\eta}_{1}, \eta_{3} \rightarrow(5.2) \\ \bar{\eta}_{2}, \eta_{2} \rightarrow(5.3)}} .
$$

\footnotetext{
${ }^{18}$ Due to translational invariance, Fourier transforming in both $x_{1}$ and $x_{3}$ will lead to a divergent answer. This can be fixed by using Gaussian wavepackets and taking the limit of plane waves in the end. Alternatively we can simply keep the third point fixed and integrate in $x_{13}$ only.

${ }^{19}$ The conventions are

$$
x^{+}=x^{0}+x^{3}=\boldsymbol{\xi}_{-} \times \overline{\boldsymbol{\xi}}_{+}, \quad x^{-}=x^{0}-x^{3}=\boldsymbol{\xi}_{+} \mathrm{x} \overline{\boldsymbol{\xi}}_{-}, \quad x^{2}=-x^{+} x^{-}+\vec{x}_{\perp}^{2} .
$$
}


In order to enforce the correct ordering, the integral in $y^{-}$must be supplemented with the appropriate $i \epsilon$ prescription, namely $y^{0} \rightarrow y^{0}-i \epsilon$ and $x^{0} \rightarrow x^{0}-2 i \epsilon$. The integrals and the limit $y^{+} \rightarrow \infty$ remove all dependence on the points $x, y$. The result is therefore a polynomial in the variables $p, m, \bar{p}$ and $\bar{m}$. The same considerations apply for the norm of the state, which is computed by Fourier transforming the two-point function

$$
\left.\mathcal{F}\left[\mathrm{n}_{\bar{O} O}\right] \equiv \int_{\mathbb{R}^{4}} \mathrm{~d}^{4} x e^{-i x^{0}} \mathrm{n}_{\bar{O} O}\left(x ; \bar{\eta}_{1}, \eta_{3}\right)\right|_{\bar{\eta}_{1}, \eta_{3} \rightarrow(5.2)} .
$$

The restrictions imposed by $\mathrm{SO}(2)$ invariance imply that only certain terms can appear, i.e.

$$
\mathcal{A}\left[\mathrm{t}_{\bar{O} T O}\right]=\sum_{s=0}^{j} \mathcal{A}_{s}\left[\mathrm{t}_{\bar{O} T O}\right](p \bar{m})^{s}(m \bar{p})^{j-s}, \quad \mathcal{F}\left[\mathrm{n}_{\bar{O} O}\right]=\sum_{s=0}^{j} \mathcal{F}_{s}\left[\mathrm{n}_{\bar{O} O}\right](p \bar{m})^{s}(m \bar{p})^{j-s} .
$$

Each coefficient of this polynomial corresponds to a different choice for the polarizations of $O$ and $\bar{O}$, therefore the polarization matrix is diagonal and the ANEC states

$$
\mathcal{E}[\Delta ;(j, 0) ; s] \equiv \frac{\mathcal{A}_{s}\left[\mathrm{t}_{\bar{O} T O}\right]}{\mathcal{F}_{s}\left[\mathrm{n}_{\bar{O} O}\right]} \geqslant 0, \quad \text { for } s=0, \ldots, j .
$$

The integrals have been computed explicitly for some values of $j$ in [10]. Here we provide a general formula, whose proof can be found in appendix B:

$$
\begin{aligned}
\mathcal{E}[\Delta ;(j, 0) ; s]=\frac{3 \pi(-i)^{j}}{8} \frac{(\delta-1)(\delta+j)}{(\delta+j-s-1)_{3}}\left(D_{1}\right. & +\frac{j-s}{j} \frac{\delta+j-1}{\delta+j-s-2} D_{2} \\
& \left.+\frac{(j-s-1)_{2}}{(j-1)_{2}} \frac{(\delta-j-2)_{2}}{(\delta+j-s-3)_{2}} D_{3}\right),
\end{aligned}
$$

where $\delta=\Delta-\frac{1}{2} j-1$ and $(a)_{n}=\Gamma(a+n) / \Gamma(a)$ is the Pochhammer symbol. See table 5 for the meaning of the three-point function coefficients. For the special cases $j=0,1$ it suffices to set to zero the absent coefficient(s). Note that (5.9) is real because the coefficients $D_{i}$ are purely real (resp. imaginary) if $j$ is even (resp. odd).

\subsection{ANEC on a superposition of states}

In the previous subsection the operator $O$ could have been either the superconformal primary or the first superdescendant $Q O^{ \pm}$. However, these operators mix with each other, i.e. the three-point function $\left\langle\left(\bar{Q} \bar{O}^{+}\right) T\left(Q O^{-}\right)\right\rangle$is nonzero. This means that we can impose an even stronger constraint by demanding positivity on the general superposition

$$
|\psi\rangle=\frac{v\left(Q O^{+}\right)|0\rangle}{\left|\left\langle\left(\bar{Q} \bar{O}^{+}\right)\left(Q O^{+}\right)\right\rangle\right|^{1 / 2}}+\frac{w\left(Q O^{-}\right)|0\rangle}{\left|\left\langle\left(\bar{Q} \bar{O}^{-}\right)\left(Q O^{-}\right)\right\rangle\right|^{1 / 2}} .
$$

A similar approach was used in [32]. Since $v$ and $w$ can be chosen arbitrarily, the ANEC now becomes a semidefinite-positiveness constraint on a $2(j+1) \times 2(j+1)$ matrix. Such a matrix can be decomposed in $j$ blocks of size $2 \times 2$ and two $1 \times 1$ blocks, resulting in

$$
\begin{aligned}
\left(\begin{array}{ll}
\mathcal{E}\left[\Delta+\frac{1}{2} ;(j+1,0) ; s+1\right] & \mathcal{E}_{\text {int }}\left[\Delta+\frac{1}{2} ;(j \pm 1,0) ; s\right] \\
\mathcal{E}_{\text {int }}\left[\Delta+\frac{1}{2} ;(j \pm 1,0) ; s\right] & \mathcal{E}\left[\Delta+\frac{1}{2} ;(j-1,0) ; s\right]
\end{array}\right) \succeq 0 & \text { for } s=0, \ldots, j-1, \\
\mathcal{E}\left[\Delta+\frac{1}{2} ;(j+1,0) ; s\right] & \geqslant 0 \quad \text { for } s=0, j+1 .
\end{aligned}
$$


The diagonal entries have the same expression as (5.9) with the substitution $D_{i} \rightarrow J_{i}$ or $D_{i} \rightarrow M_{i}$ (see tables 20,23), together with the appropriate redefinition of $\delta$. The "interference" terms $\mathcal{E}_{\text {int }}$ are defined as follows: ${ }^{20}$

$$
\begin{aligned}
\mathcal{E}_{\text {int }}\left[\Delta+\frac{1}{2} ;(j+1,0) ; s\right] & =\mathcal{E}_{\text {int }}\left[\Delta+\frac{1}{2} ;(j-1,0) ; s\right] \\
& \equiv \frac{\mathcal{A}_{s}\left[\mathrm{t}_{\left(\bar{Q} \bar{O}^{+}\right) T\left(Q O^{-}\right)}\right]}{\left(\mathcal{F}_{s+1}\left[\mathrm{n}_{\left(\bar{Q} \bar{O}^{+}\right)\left(Q O^{+}\right)}\right] \mathcal{F}_{s}\left[\mathrm{n}_{\left(\bar{Q} \bar{O}^{-}\right)\left(Q O^{-}\right)}\right]\right)^{1 / 2}} .
\end{aligned}
$$

Following steps similar to the ones illustrated in appendix B one can prove the general formula

$$
\begin{aligned}
\mathcal{E}_{\text {int }}\left[\Delta+\frac{1}{2} ;(j \pm 1,0) ; s\right]= & \frac{3 \pi(-i)^{j-1}}{16} \sqrt{\frac{\delta(s+1)(j-s)}{j(j+1)(\delta+j+1)}} \frac{(\delta+j-1)_{3}}{(\delta+j-s-2)_{4}} \\
& \times\left(\frac{\delta+j-s-2}{\delta+j-1} K_{1}+\frac{j-s-1}{j-1} K_{2}\right)
\end{aligned}
$$

where the coefficients $K_{i}=L_{i}$ are defined in tables 21, 22 and $\delta=\Delta_{Q O}-\frac{1}{2} j-\frac{3}{2}$. Here $\Delta_{Q O}=\Delta+\frac{1}{2}$ is the dimension of the superdescendant. The polarization $s$ takes values from 0 to $j-1$.

\subsection{Operators of $\operatorname{spin}\left(\frac{1}{2} j, \frac{1}{2}\right)$}

The only difference when considering more general $\mathrm{SO}(1,3)$ representations is that the polarization matrix will not be diagonal. This means that the ANEC will not be a set of simple inequalities but rather semidefinite positiveness constraints. In the $\left(\frac{1}{2} j, \frac{1}{2}\right)$ case we further have to specify the polarizations $\eta_{1}$ and $\bar{\eta}_{3}$; thus together with (5.2) and (5.3) one has

$$
\eta_{1}^{\alpha}=\left(\begin{array}{c}
m^{\prime} \\
p^{\prime}
\end{array}\right) \equiv m^{\prime} \boldsymbol{\xi}_{-}^{\alpha}+p^{\prime} \boldsymbol{\xi}_{+}^{\alpha}, \quad \bar{\eta}_{3}^{\dot{\alpha}}=\left(\begin{array}{c}
\bar{p}^{\prime} \\
\bar{m}^{\prime}
\end{array}\right) \equiv \bar{p}^{\prime} \overline{\boldsymbol{\xi}}_{+}^{\dot{\alpha}}+\bar{m}^{\prime} \overline{\boldsymbol{\xi}}_{-}^{\dot{\alpha}}
$$

The ANEC integral for an arbitrary operator $O$ of spin $\left(\frac{1}{2} j, \frac{1}{2}\right)$ takes the form

$$
\left.\tilde{\mathcal{A}}\left[\mathrm{t}_{\bar{O} T O}\right] \equiv \int_{-\infty}^{\infty} \mathrm{d} y^{-} \lim _{y^{+} \rightarrow \infty} \frac{\left(y^{+}\right)^{2}}{16} \int_{\mathbb{R}^{4}} \mathrm{~d}^{4} x e^{-i x^{0}} \mathrm{t}_{\bar{O} T O}\left(x, y ; \eta_{1,2,3}, \bar{\eta}_{1,2,3}\right)\right|_{\begin{array}{l}
\bar{\eta}_{1}, \eta_{3} \rightarrow(5.2) \\
\overline{\bar{\eta}}_{3}, \eta_{1} \rightarrow(5.13) \\
\bar{\eta}_{2}, \eta_{2} \rightarrow(5.3)
\end{array}}
$$

We also define $\tilde{\mathcal{F}}\left[\mathrm{n}_{\bar{O} O}\right]$ in a similar way. The constraints of $\mathrm{SO}(2)$ invariance allow us to express

$$
\tilde{\mathcal{A}}\left[\mathrm{t}_{\bar{O} T O}\right]=\sum_{s=0}^{j+1} \sum_{a, b=0}^{1}\left(\tilde{\mathcal{A}}_{s}\left[\mathrm{t}_{\bar{O} T O}\right]\right)_{a b}(p \bar{m})^{s}(m \bar{p})^{j-s} p^{\prime} \bar{m}^{\prime}\left(\frac{\bar{p} m^{\prime}}{\bar{m} p^{\prime}}\right)^{a}\left(\frac{m \bar{p}^{\prime}}{p \bar{m}^{\prime}}\right)^{b},
$$

\footnotetext{
${ }^{20}$ The definition of $\mathcal{A}_{s}$ for the interference correlator is similar to (5.6) with the difference that we pick up the term $\bar{m} \bar{p}(p \bar{m})^{s}(m \bar{p})^{j-s-1}$ for $\left\langle\left(\bar{Q} \bar{O}^{+}\right) T\left(Q O^{-}\right)\right\rangle$and $m p(p \bar{m})^{s}(m \bar{p})^{j-s-1}$ for its conjugate.
} 
and similarly for $\tilde{\mathcal{F}}\left[\mathrm{n}_{\bar{O} O}\right]$. The terms for $s=0$ and $s=j+1$ are restricted to, respectively, $a=b=0$ and $a=b=1$. Thus we can see that the polarization matrix is block diagonal with $j$ blocks of size $2 \times 2$ and two blocks of size $1 \times 1$. Defining

$$
(\mathcal{E}[\Delta ;(j, 1) ; s])_{a b} \equiv \frac{\left(\tilde{\mathcal{A}}_{s}\left[\mathrm{t}_{\bar{O} T O}\right]\right)_{a b}}{\left.\left(\left(\tilde{\mathcal{F}}_{s}\left[\mathrm{n}_{\bar{O} O}\right]\right)_{a a} \tilde{\mathcal{F}}_{s}\left[\mathrm{n}_{\bar{O} O}\right]\right)_{b b}\right)^{1 / 2}},
$$

the positivity constraints are

$$
\begin{array}{ll}
\mathcal{E}[\Delta ;(j, 1) ; s] \succeq 0, & \text { for } s=1, \ldots, j, \\
\mathcal{E}[\Delta ;(j, 1) ; s] \geqslant 0, & \text { for } s=0, j+1 .
\end{array}
$$

In the next subsection we will explain how to implement a numerical study of this system of inequalities. We obtained a general formula for $\mathcal{E}[\Delta ;(j, 1) ; s]$ as well - unfortunately, however, the expression is too unwieldy to be reported here. In appendix B we briefly explain how to obtain it.

\subsection{The ANEC as a semidefinite programming problem}

Imposing semidefinite positiveness on a symmetric matrix is a well known problem for which there exist algorithms that go under the name of semidefinite programming. We will make use of the implementation realized by the software sdpb [23], which was developed for the numerical bootstrap approach for the study of CFTs [33], but is general purpose enough to work for our problem too.

In general we need to solve a system of inequalities

$$
\mathcal{E}[\Delta ;(j, \bar{\jmath}) ; s] \succeq 0, \quad \text { for } s=0, \ldots, j+\bar{\jmath},
$$

where $\mathcal{E}[\Delta ;(j, \bar{\jmath}) ; s]$ is a symmetric $m_{s} \times m_{s}$ matrix with $m_{s}=\min \{j, \bar{\jmath}, s, j+\bar{\jmath}-s\}+1$. The matrices $\mathcal{E}$ will depend on $N$ arbitrary three-point function coefficients (given by table 1) plus an inhomogeneous part which is fixed by the Ward identities. Dropping the $\Delta$ and $(j, \bar{\jmath})$ labels for brevity one has

$$
\mathcal{E}[s]=\mathcal{E}^{(0)}[s]+\sum_{n=1}^{N} \lambda_{n} \mathcal{E}^{(n)}[s] \succeq 0, \quad \text { for } s=0, \ldots, j+\bar{\jmath} .
$$

This is known as the dual formulation of a semidefinite problem. We are interested in studying the feasibility of (5.19). The algorithm we used only terminates when either a solution $\lambda_{n}$ is found, or when a numerical threshold for the internal computations ${ }^{21}$ is exceeded. For our purposes, a problem that terminates for the latter condition is considered to have no solution. This means that our ANEC-disallowed points are not disallowed in a mathematically rigorous way. We expect this to not have any practical consequences. ${ }^{22}$

\footnotetext{
${ }^{21}$ Called --maxComplementarity.

${ }^{22}$ In principle there is also a way to mathematically prove that no solutions exist by providing a certificate of infeasibility [34]. By using [35-37] this amounts to finding a solution of another (larger) semidefinite problem.
} 

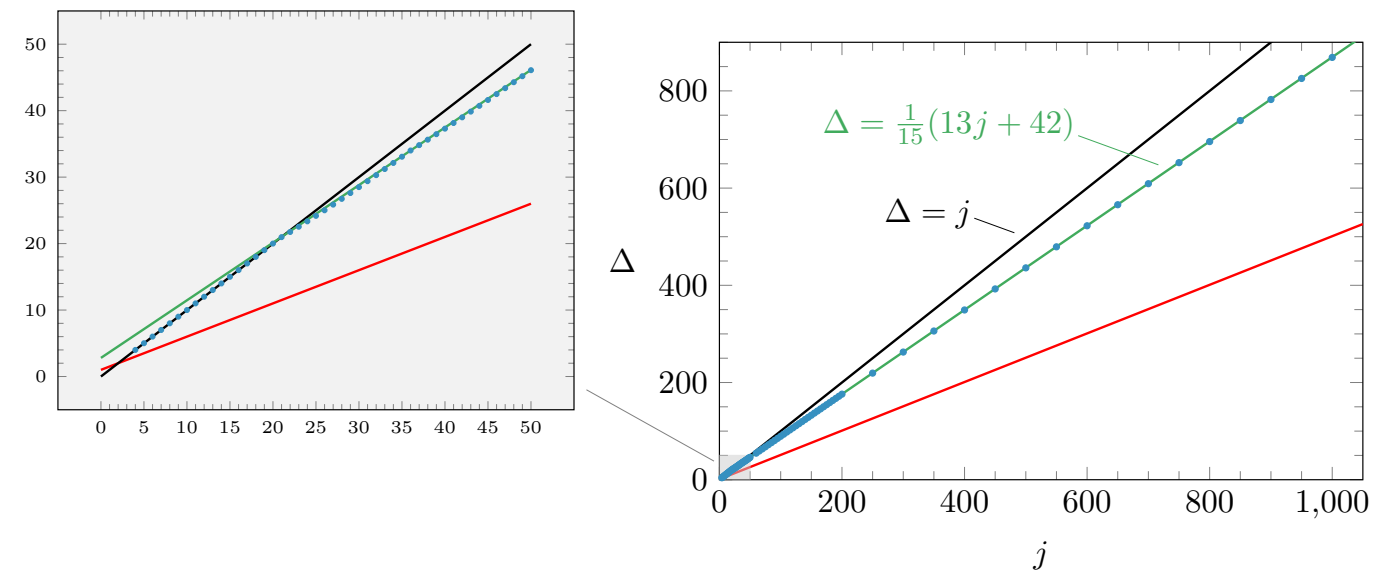

Figure 5. Lower bounds on the conformal dimension $\Delta$ as a result of the ANEC for primaries transforming in the $\left(\frac{1}{2} j, 0\right)$ Lorentz representation. Each point is the result of a bisection in $\Delta$. The red line is the unitarity bound, $\Delta=\frac{1}{2} j+1$. The black line corresponds to the conjecture of [10], $\Delta=j$, and the green line gives an approximate behavior of the bound valid above $j=20$.

\subsection{Details on ANEC bounds: nonsupersymmetric case}

Let us briefly review the results obtained in [10] and prove a few results for generic values of $j$. First let us consider conformal primaries in the $\left(\frac{1}{2} j, 0\right)$ Lorentz representation. The ANEC condition is expressed by the formula (5.9), where the coefficients $D_{i}$ are given in table 5 . In particular, one can take $\hat{D}_{1}=-i^{j} D_{1}$ to be the only independent real coefficient. By choosing the value $s=0$ and $s=j$ in (5.9) and restricting to the case $j>2$ for simplicity we obtain

$$
(\delta-1)\left(\left(\pi^{2} \hat{D}_{1}-4\right) \delta+j\left(\pi^{2} \hat{D}_{1}+2 \delta-6\right)+2 j^{2}+4\right) \geqslant 0, \quad \hat{D}_{1} \geqslant 0,
$$

where $\delta \geqslant 0$ represents the distance from the unitarity bound. It is straightforward to verify that the above conditions cannot be simultaneously satisfied unless $\delta \geqslant 1$.

By considering all polarizations we can obtain stronger bounds at the price of fixing the value of $j$, for instance by using the function Reduce of Mathematica. We show our results in figure 5 up to $j=10^{3}$. Although the bound initially agrees with the conjecture of [10], it departs from it for $j \geqslant 21$ and follows a different pattern which is well fitted by the expression $\Delta=\frac{1}{2} j+1+\delta \geqslant \frac{1}{15}(13 j+42)$. It would be tempting to assign a meaning to the kink at $j \sim 21$, but the explanation might simply reside in the fact that, going to large values, the integer nature of $j$ becomes less and less important and new solutions for $\hat{D}_{1}$ become available.

Let us now move to the case of conformal primaries in the $\left(\frac{1}{2} j, \frac{1}{2}\right)$ representation. The procedure to obtain the general formula is described in appendix B.2. After imposing the Ward identities, whose solution is reported in table 6 , one is left with four independent three-point function coefficients $H_{i}$. In order to systematically address the feasibility of the ANEC we translated the linear matrix inequality into a semidefinite problem as discussed in the previous subsection. We found agreement with the results of [10] for $j \leqslant 7$ and 

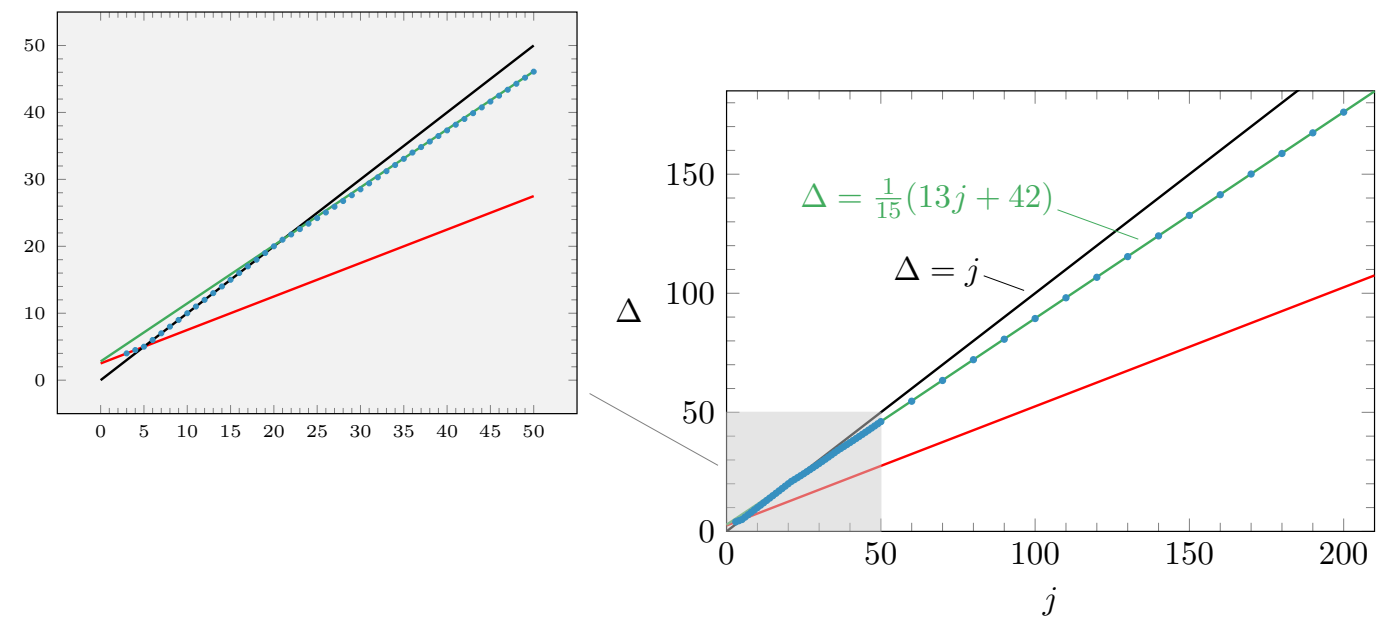

Figure 6. Lower bounds on the conformal dimension $\Delta$ as a result of the ANEC for primaries transforming in the $\left(\frac{1}{2} j, \frac{1}{2}\right)$ Lorentz representation. Each point is the result of a bisection in $\Delta$. The red line is the unitarity bound, $\Delta=\frac{1}{2} j+\frac{5}{2}$. The operators for $j \leqslant 5$ lie on the red line. The black line corresponds to the conjecture of [10], $\Delta=j$, and the green line gives an approximate behavior of the bound valid above $j=20$.

extended the bounds up to $j=50$. A lower bound on $\Delta$ as a function of $j$ is shown in figure 6: again we observe that for $j>21$ the bounds departs from the conjecture $\Delta \geqslant j$ of [10] and closely follows the bound $\Delta \geqslant \frac{1}{15}(13 j+42)$ instead.

In the case of conserved operators the problem simplifies considerably: only two coefficients remain independent ${ }^{23}$ and we can easily prove that conserved currents cannot exist for $j>5$. For instance, we can take $\hat{H}_{9,10}=-i^{j+1} H_{9,10}$ to be the two independent real coefficients. By considering the eigenvalues of matrices with $s=j-3, \ldots, j$ and the condition at $s=j+1$, we obtain the following set of inequalities:

$$
\begin{aligned}
\hat{H}_{10} & \geqslant 0, & 3 \hat{H}_{9}+\frac{18}{\pi^{2}} \frac{j-1}{j+1} & \leqslant \hat{H}_{10} \frac{2 j+1}{j-1}, \\
\hat{H}_{9} & \leqslant \frac{2}{3} \hat{H}_{10}, & 3 \hat{H}_{9}+\frac{12}{\pi^{2}} & \geqslant 2 \hat{H}_{10} \frac{j+1}{j-1} .
\end{aligned}
$$

One can immediately check that the above conditions admit a solution only for $j \leqslant 5$, corresponding to the cases when conserved currents can be constructed in free theories. Interestingly, for the boundary case $j=5$ the solution to the ANEC is unique:

$$
\hat{H}_{9}=-\frac{4}{\pi^{2}}, \quad \hat{H}_{10}=0 .
$$

\subsection{Details on ANEC bounds: supersymmetric case}

In the supersymmetric case the analysis follows the same steps as before, except that now one needs to combine multiple conditions. Let us discuss some of the results presented

\footnotetext{
${ }^{23}$ The relation imposed by conservation of the operator $O$ can be easily computed using the package CFTs4D.
} 
in the introduction. We first start from a multiplet whose zero component transforms in the $\left(\frac{1}{2} j, 0\right)$ representation and satisfies the $[L, \bar{B}]$ shortening condition. These are the generalizations to $j>1$ of the usual chiral scalar and gauge-invariant spin- $\frac{1}{2}$ multiplets. In this case $\bar{q}=0$ and $q=\Delta$. The multiplet contains only four conformal primaries: $O, Q O^{ \pm}$ and $Q^{2} O$. In this work we only consider the first three. As discussed in section 3.4 the superspace three-point function does not have any free parameters. Let us consider, then, the ANEC applied to the superprimary only. The condition is again encoded in (5.9), where now the coefficients $D_{i}$ are related to the superspace coefficients through the relations in table 11 , supplemented by the relations in table 2 . The analog of $\hat{D}_{1} \geqslant 0$ in (5.20) is now simply

$$
2 q-3 j \geqslant 0
$$

We explicitly checked that including other constraints does not strengthen the bound. This is expected since one can construct chiral operators with $\Delta=\frac{3}{2} j$ by taking products of free chiral vector multiplets. The bound is therefore optimal.

Let us move to another simple case, namely $\left[A_{1}, \bar{A}_{2}\right]$, corresponding to superprimaries again in the $\left(\frac{1}{2} j, 0\right)$ representation with $q=\frac{1}{2} j+1$ and $\bar{q}=1$. This multiplet contains conserved operators in the $\left(\frac{1}{2}(j+1), \frac{1}{2}\right)$ and, due to the results of the previous subsection, we can immediately conclude that $j \geqslant 4$. It turns out, however, that $j=4$ is excluded since the values $\hat{H}_{9}$ and $\hat{H}_{10}$ fixed by supersymmetry do not satisfy (5.22). Smaller values of $j$ must be consistent since these operators appear in the decomposition of extended supersymmetry multiplets in the free limit.

All other bounds found in this work were obtained with a numerical approach. For completeness we collect here all the conditions we imposed in the most complicated case $[L, \bar{L}]$. In simpler cases some of them do not appear since the corresponding superdescendant is absent. At the same time, the correct three-point function coefficient relations must be imposed. Given an $[L, \bar{L}]$ supermultiplet with a superprimary transforming in the $\left(\frac{1}{2} j, 0\right)$ representation and $q \geqslant \frac{1}{2} j+1, \bar{q} \geqslant 1$, the ANEC can be satisfied if there exist real coefficients $\hat{\mathcal{C}}_{k}=i^{j} \mathcal{C}_{k}, k=2,6$, such that

$$
\begin{aligned}
& \langle\bar{O} T O\rangle: \\
& \mathcal{E}[\Delta,(j, 0) ; s] \geqslant 0, \quad \text { for } s=0, \ldots, j, \\
& \langle(\bar{Q} \bar{O}) T(Q O)\rangle \text { : } \\
& \left(\begin{array}{lr}
\mathcal{E}\left[\Delta+\frac{1}{2} ;(j+1,0) ; s+1\right] & \mathcal{E}_{\text {int }}\left[\Delta+\frac{1}{2} ;(j \pm 1,0) ; s\right] \\
\mathcal{E}_{\text {int }}\left[\Delta+\frac{1}{2} ;(j \pm 1,0) ; s\right] & \mathcal{E}\left[\Delta+\frac{1}{2} ;(j-1,0) ; s\right]
\end{array}\right) \succeq 0 \quad \text { for } s=0, \ldots, j-1, \\
& \mathcal{E}\left[\Delta+\frac{1}{2} ;(j+1,0) ; s\right] \geqslant 0 \quad \text { for } s=0, j+1, \\
& \langle(Q \bar{O}) T(\bar{Q} O)\rangle \text { : } \\
& \mathcal{E}\left[\Delta+\frac{1}{2} ;(j, 1) ; s\right] \succeq 0, \quad \text { for } s=0, \ldots, j+1 .
\end{aligned}
$$


As usual we defined $\Delta=q+\bar{q}$. Whenever the above system of conditions does not admit a solution, we conclude that the corresponding supersymmetry multiplet cannot exist in a local unitary SCFT.

\section{Bounds on extended supersymmetry multiplets}

\subsection{Conventions}

The aim of this section is to constrain the superconformal multiplets of theories with $\mathcal{N}>1$ supersymmetry by decomposing them into $\mathcal{N}=1$ multiplets. This approach does not make use of the additional linear relations among the three-point function coefficients and thus may not yield optimal bounds. Following [19], we will denote $\mathcal{N}=2$ supermultiplets as $\mathcal{X}_{L} \overline{\mathcal{X}}_{R}[j, \bar{\jmath}]_{\Delta}^{(\mathcal{R}, r)}$, where $(\mathcal{R}, r)$ are the quantum numbers under the $\mathfrak{s u}(2) \oplus \mathfrak{u}(1)$ algebra, while we will denote $\mathcal{N}=4$ supermultiplets as $\mathcal{X}_{L} \overline{\mathcal{X}}_{R}[j, \bar{j}]_{\Delta}^{\left(p_{1}, p_{2}, p_{3}\right)}$, where $p_{1}, p_{2}$ and $p_{3}$ are the Dynkin labels of the $\mathfrak{s u}(4)$ algebra representation $\left[p_{1}, p_{2}, p_{3}\right]$, for which we use the conventions of [38]. As in previous sections, the left/right shortening can take values $\mathcal{X}_{L, R}=L, A_{1}, A_{2}, B_{1}$.

We define the supercharges to transform under the $\mathfrak{u}(1)$ R-symmetry of the $\mathcal{N} \neq 4$ superalgebra as

$$
\left[r_{\mathcal{N}}, Q_{\alpha}^{I}\right]=-Q_{\alpha}^{I}, \quad\left[r_{\mathcal{N}}, \bar{Q}_{I \dot{\alpha}}\right]=\bar{Q}_{I \dot{\alpha}}
$$

We consider for any $\mathcal{N}$ the superalgebra generated by $Q_{\alpha}^{1}$ and $\bar{Q}_{1 \dot{\alpha}}$. The embedding of the $\mathcal{N}=1 \mathfrak{u}(1)$ R-charge in the larger R-symmetry group is

$$
\begin{array}{ll}
\mathcal{N}=2: & R \equiv r_{\mathcal{N}=1}=-\frac{4}{3} R_{3}+\frac{1}{3} r_{\mathcal{N}=2}, \\
\mathcal{N}=4: & R \equiv r_{\mathcal{N}=1}=-\frac{1}{3}\left(3 H_{1}+2 H_{2}+H_{3}\right)
\end{array}
$$

where $H_{i}$ is the Cartan generator associated to the $i$-th Dynkin label in $\left[p_{1}, p_{2}, p_{3}\right]$. The generator $R_{3}$ is the $\mathfrak{s u}(2)$ Cartan in units of $\frac{1}{2}\left(R_{3}=-\frac{1}{2} \mathcal{R}, \ldots, \frac{1}{2} \mathcal{R}\right)$. Consistently with the rest of the paper, $R$ is the $\mathcal{N}=1$ R-charge. We will also abbreviate $r \equiv r_{\mathcal{N}=2}$.

\section{$6.2 \mathcal{N}=2$}

Let us start by considering the so-called "exotic chiral primaries," namely the $L \bar{B}_{1}[j ; 0]_{\Delta}^{(0, r)}$ multiplets, with $\Delta=\frac{1}{2} r{ }^{24}$ The bound on chiral multiplets (1.8) for the $\mathcal{N}=1$ subalgebra generated by $Q_{\alpha}^{1}$, applied to the chiral superprimary $Q_{\left(\alpha_{1}\right.}^{2} \mathcal{O}_{\left.\alpha_{2} \ldots \alpha_{j+1}\right)}^{(\text {exotic })}$ implies that

$$
\Delta+\frac{1}{2} \geqslant \frac{3}{2}(j+1) \quad \Rightarrow \quad \Delta \geqslant \frac{3}{2} j+1
$$

The unitarity bound is $\Delta \geqslant \frac{1}{2} j+1$, and so we see that the ANEC bound is stronger for $j>0$.

\footnotetext{
${ }^{24}$ Denoted $\overline{\mathcal{E}}_{\frac{r}{2}(j, 0)}$ in $[38]$.
} 
A similar argument can be made on operators with nonzero $\mathfrak{s u}(2)$ R-charge $L \bar{B}_{1}[j ; 0]_{\Delta}^{(\mathcal{R}, r)}$, where $\Delta=\mathcal{R}+\frac{1}{2} r$ and $\mathcal{R}$ is in integer units. We considered several values of $\mathcal{R}$ and performed the decomposition into $\mathcal{N}=1$ multiplets. Imposing (1.8) on each of the chiral multiplets that appear yields the following pattern (which we conjecture to be true for arbitrary $\mathcal{R}$ ):

$$
r \geqslant 3 j+2-2 \mathcal{R} \quad \Rightarrow \quad \Delta \geqslant \frac{3}{2} j+1 .
$$

This is stronger than unitarity $(r \geqslant j+2)$ for $j>\mathcal{R}$. As a consequence, short multiplets of the form $A_{\ell} \bar{B}_{1}[j ; 0]_{\Delta}^{(\mathcal{R}, r)}$ are only allowed for $j \leqslant \mathcal{R}$.

The multiplets $A_{1} \bar{B}_{1}[j ; 0]_{\Delta}^{(1, j+2)}$ and $A_{1} \bar{A}_{2}[j ; 0]_{\Delta}^{(0, j)}$ with $\Delta=\frac{1}{2} j+2$ are absent from any local SCFT for $j>2$. This is a consequence of the presence of an $A_{1} \bar{A}_{2}[j+1 ; 0]$ multiplet in their $\mathcal{N}=1$ decomposition, which we have shown to be forbidden by the ANEC when $j+1>3$.

We also considered long multiplets $L \bar{L}[j ; 0]_{\Delta}^{(\mathcal{R}, r)}$ for some values of $\mathcal{R}$. Calling $\delta$ the difference of their dimension and their unitarity bound,

$$
\delta=\Delta-2-j-\mathcal{R}+\frac{1}{2} r
$$

and calling $f(R, j)$ the separation between the unitarity and the ANEC bound in figure 2, we find the following pattern

$$
\delta \geqslant f\left(\frac{1}{3}(r+1), j+1\right)-\mathcal{R} .
$$

\section{$6.3 \mathcal{N}=4$}

We considered a few short multiplets and found no constraints from the ANEC. Interestingly, $B_{1} \bar{B}_{1}[0 ; 0]_{2}^{(1,0,1)}$ contains a chiral multiplet that saturates (1.8), namely

$$
B_{1} \bar{B}_{1}[0 ; 0]_{2}^{(1,0,1)} \supset L \bar{B}_{1}[2 ; 0]_{3}^{(2)} .
$$

The simplest long multiplet is the Konishi multiplet $L \bar{L}[0 ; 0]^{(0,0,0)}$. In its $\mathcal{N}=1$ decomposition we find a long multiplet of spin $\left(\frac{3}{2}, 0\right)$ and R-charge 1 with dimension $\Delta_{\text {Konishi }}+\frac{3}{2}$. In terms of the $Q_{\alpha}^{1}$ subalgebra, calling $\phi$ the Konishi operator, one has

$$
\mathcal{O}_{\alpha_{1} \alpha_{2} \alpha_{3}}=\varepsilon_{1 I J K} Q_{\left(\alpha_{1}\right.}^{I} Q_{\alpha_{2}}^{J} Q_{\left.\alpha_{3}\right)}^{K} \phi
$$

Since in perturbation theory one can compute $\Delta_{\text {Konishi }}=2+O\left(g^{2}\right)$, we see that the ANEC and the unitarity bound for $\mathcal{N}=1$ long multiplets of $\operatorname{spin}\left(\frac{3}{2}, 0\right)$ are saturated.

More generally, we checked some cases of long multiplets $L \bar{L}[j ; 0]^{\left(p_{1}, p_{2}, p_{3}\right)}$, namely those with Dynkin labels $\left[p_{1}, p_{2}, p_{3}\right]=[0,0,0],[0,2,0]$ and $[1,0,1]$. Calling $\delta$ the difference of their dimension and their unitarity bound,

$$
\delta=\Delta-2-j-\frac{1}{2}\left(3 p_{1}+2 p_{2}+p_{3}\right),
$$


and calling $f(R, j)$ the separation between the unitarity and the ANEC bound in figure 2, we find

$$
\begin{array}{ll}
{[0,0,0]:} & \delta \geqslant f\left(\frac{4}{3}, j+2\right)-2, \\
{[0,2,0]:} & \delta \geqslant f\left(\frac{7}{3}, j+3\right)-4, \\
{[1,0,1]:} & \delta \geqslant f\left(\frac{7}{3}, j+3\right)-4 .
\end{array}
$$

\section{Conclusions and outlook}

In this paper we studied effects of the ANEC on the operator spectrum of CFTs. In particular, we showed that the ANEC imposes lower bounds on operator dimensions that are stronger than unitarity bounds. Our considerations were mostly limited to the case of $\mathcal{N}=1$ superconformal multiplets whose superconformal primaries transform in the $\left(\frac{1}{2} j, 0\right)$ representation of the Lorentz group. This suffices to show that the unitarity bounds are typically suboptimal to the ANEC bounds.

Our methods apply in more general situations, with or without supersymmetry. It would be of great value to obtain an educated guess for the ANEC bound on multiplets whose superconformal primaries transform in the general $\left(\frac{1}{2} j, \frac{1}{2} \bar{\jmath}\right)$ representation. In this respect, the techniques presented here to compute the ANEC integral in closed form and the usage of semidefinite programming will considerably simplify the analysis.

These ideas can also be generalized to extended supersymmetry, in particular $\mathcal{N}=2$. In principle it is possible to carry out a similar analysis for the three-point functions in $\mathcal{N}=2$ superspace with a formalism similar to the one used in this paper and using results of $[39,40]$. One of the motivations behind pursuing this direction would be to potentially further constrain the exotic chiral primaries $L \bar{B}_{1}[j ; 0]_{r / 2}^{(0, r)}$. These operators for $j \geqslant 1$ have been proved to be absent in a very large class of theories [22]. Using the results in section 6 we are able to constrain their dimension to

$$
\Delta_{\text {exotic }} \geqslant \frac{3}{2} j+1
$$

It would be interesting to see if ANEC forbids them in general once the $\mathcal{N}=2$ superconformal symmetry is fully taken into account.

In $\mathcal{N}=2$ one could also investigate the higher-spin version of the ANEC mentioned in the introduction $[7,8]$. In a generic CFT it is hard to address such a problem because, unlike the spin-two case, the dimension of the lowest-twist operator is not fixed and there are no Ward identities to constrain the three-point function coefficients. In $\mathcal{N}=2$ SCFTs, however, there are higher-spin operators with protected dimensions that are not at the unitarity bound (hence do not decouple from the theory [41, 42]). An example are the $A_{1} \bar{A}_{1}[\ell ; \ell]_{\Delta}^{(R, 0)}$ multiplets, with $\Delta=\ell+2+R$ and $R>0$. Clearly the bounds obtained this way will not be general but will assume that $R$ is the smallest $\mathrm{R}$-charge among these protected operators and, at spin $\ell$, the unprotected spectrum has a gap larger than $\ell+2+R$. We leave these questions for future investigations. 


\section{Acknowledgments}

This work was initiated at the Bootstrap 2018 conference held at Caltech. We thank the organizers for creating a stimulating atmosphere, and the participants for interesting conversations. We especially thank Clay Cordova for inspiring discussions about his work. We also thank Kenan Diab, Zohar Komargodski and Leonardo Rastelli for useful comments. Finally, we thank Ning Su for helpful comments about the performance of sdpb. $\mathrm{AM}$ and AV are supported by the Swiss National Science Foundation under grant no. PP00P2-163670. AV is also supported by the European Research Council Starting Grant under grant no. 758903. Some of the computations in this paper were run on the EPFL SCITAS cluster.

\section{A Supersymmetric inversion tensors}

Here we list the properties needed to derive equation (3.10). The order in which they appear is roughly the order in which one needs to apply them. First of all, the explicit definition of the tensors is

$$
\begin{aligned}
& I_{\mu \nu}\left(x_{1 \overline{2}}, x_{\overline{1} 2}\right)=\bar{I}_{\nu \mu}\left(x_{\overline{2} 1}, x_{2 \overline{1}}\right)=\frac{\operatorname{tr}\left(\sigma_{\mu} \tilde{\mathrm{x}}_{\overline{1} 2} \sigma_{\nu} \tilde{\mathrm{x}}_{\overline{2} 1}\right)}{2 \sqrt{x_{\overline{1} 2}{ }^{2} x_{\overline{2} 1}{ }^{2}}}=\frac{\operatorname{tr}\left(\bar{\sigma}_{\mu} \mathrm{x}_{1 \overline{2}} \bar{\sigma}_{\nu} \tilde{\mathrm{x}}_{2 \overline{1}}\right)}{2 \sqrt{x_{\overline{1} 2}{ }^{2} x_{\overline{2} 1}{ }^{2}}},
\end{aligned}
$$

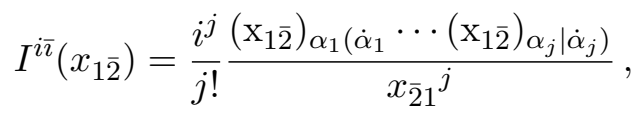

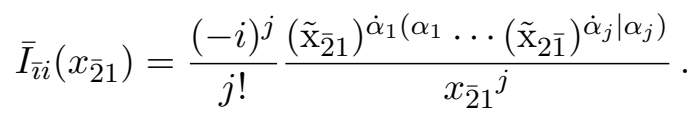

The needed properties are

$$
\begin{aligned}
I_{\mu \nu}(x, \bar{x}) \bar{I}^{\nu \rho}(-x,-\bar{x}) & =\delta_{\mu}^{\rho}, \\
I^{i \bar{\imath}}(x) \bar{I}_{\bar{\imath} i^{\prime}}(-x) & =\delta_{i^{\prime}}^{i} \\
I_{\lambda \rho}\left(x_{1 \overline{3}}, x_{\overline{1} 3}\right) \bar{I}^{\rho \nu}\left(x_{\overline{3} 2}, x_{3 \overline{2}}\right) I_{\nu \mu}\left(x_{2 \overline{1}}, x_{\overline{2} 1}\right) & =I_{\lambda \mu}\left(-\bar{X}_{1},-X_{1}\right) .
\end{aligned}
$$

The covariance property of the $t$ and its $\lambda \bar{\lambda}$ scaling (2.8) imply

$$
\begin{aligned}
I^{i_{1} \bar{\imath}_{1}}\left(x_{1 \overline{3}}\right) I^{i_{4} \bar{\imath}_{3}}\left(x_{1 \overline{3}}\right) & I_{\lambda \nu}\left(x_{1 \overline{3}}, x_{\overline{1} 3}\right) t_{\bar{\imath}_{1}}{ }^{\nu} \bar{\imath}_{3}\left(Z_{3}\right) \\
& =X_{1}{ }^{3} \bar{X}_{1}{ }^{3} x_{\overline{1}}{ }^{3} x_{\overline{3} 1}{ }^{3} I^{i_{1} \bar{\imath}_{1}}\left(\bar{X}_{1}\right) I^{i_{4} \bar{\imath}_{3}}\left(\bar{X}_{1}\right) I_{\lambda \nu}\left(\bar{X}_{1}, X_{1}\right) t_{\bar{\imath}_{1}}{ }^{\nu} \bar{\imath}_{3}\left(Z_{1}\right) .
\end{aligned}
$$

The last identities that we need are

$$
X_{1}^{2}=\frac{x_{\overline{2} 3}^{2}}{x_{\overline{2} 1}^{2} x_{\overline{1} 3}^{2}}, \quad \bar{X}_{1}^{2}=\frac{x_{\overline{3} 2}^{2}}{x_{\overline{3} 1_{1}^{2} x_{\overline{1} 2}^{2}}}
$$




\section{B Proof of the general formula}

\section{B.1 Formula for the $\left(\frac{1}{2} j, 0\right)$ case}

In this section we provide a proof of the formula (5.9) which we reproduce here for convenience:

$$
\begin{aligned}
\mathcal{E}[\Delta ;(j, 0) ; s]= & \frac{\mathcal{A}_{s}\left[\mathrm{t}_{\bar{O} T O}\right]}{\mathcal{F}_{s}\left[\mathrm{n}_{\bar{O} O}\right]} \\
=\frac{3 \pi(-i)^{j}}{8} \frac{(\delta-1)(\delta+j)}{(\delta+j-s-1)_{3}}\left(\begin{array}{l}
D_{1} \\
+\frac{j-s}{j} \frac{\delta+j-1}{\delta+j-s-2} D_{2}
\end{array}\right. & \left.+\frac{(j-s-1)_{2}}{(j-1)_{2}} \frac{(\delta-j-2)_{2}}{(\delta+j-s-3)_{2}} D_{3}\right) .
\end{aligned}
$$

The first step is to realize that the dependence on $j$ and $s$ is entirely coming from the tensors $\left(\mathbb{I}^{13}\right)^{\tilde{\jmath}}$ which appear both in $\mathrm{t}_{\bar{O} T O}$ at the numerator (with $\tilde{\jmath}=j, j-1, j-2$ ) and in $\mathrm{n}_{\bar{O} O}$ at the denominator (with $\tilde{\jmath}=j$ ). Let us then expand this tensor when the polarizations are replaced as in (5.2),

$$
\begin{aligned}
\left(\mathbb{I}^{13}\right)^{\tilde{\jmath}} & =\left(\eta_{3} \mathrm{x} \bar{\eta}_{1}\right)^{\tilde{\jmath}}=\left(m \bar{p} x^{+}+p \bar{m} x^{-}+m \bar{m} \mathrm{x}_{-\dot{-}}+p \bar{p} \mathrm{x}_{+\dot{+}}\right)^{\tilde{\jmath}} \\
& =\sum_{s=0}^{\tilde{\jmath}} \sum_{r=0}^{\min (s, \tilde{\jmath}-s)}\left(\begin{array}{c}
\tilde{\jmath} \\
2 r
\end{array}\right)\left(\begin{array}{c}
\tilde{\jmath}-2 r \\
s-r
\end{array}\right)\left(\begin{array}{c}
2 r \\
r
\end{array}\right)\left(x^{-}\right)^{s-r}\left(x^{+}\right)^{\tilde{\jmath}-r-s}\left(x_{\perp}^{2}\right)^{r}(p \bar{m})^{s}(m \bar{p})^{\tilde{\jmath}-s} .
\end{aligned}
$$

We obtained this result by simply doing a double binomial expansion and using $\mathrm{x}_{-\dot{-}} \mathrm{x}_{+\dot{+}}=$ $x_{\perp}^{2} \equiv\left(x^{1}\right)^{2}+\left(x^{2}\right)^{2}$. All terms where $\mathrm{x}_{--\dot{-}}$ and $\mathrm{x}_{+\dot{+}}$ appear with different powers can be thrown away as they are not $\mathrm{SO}(2)$ neutral and there are no other invariants in the tensor structures that can compensate for them. ${ }^{25}$ The first sum is precisely the sum over polarizations, and so we can remove it and focus on one $s$ at a time. The second sum, instead, can be extended to $\sum_{r=0}^{\infty}$ since the binomial coefficients are automatically zero when $r$ is out of bounds. This fact will be useful later on.

This expansion completely takes care of the polarizations of $\mathrm{n}_{\bar{O} O}$ and of the structure $D_{1}$ of $\mathrm{t}_{\bar{O} T O}$. For the other two structures it is not hard to see that the terms $(p \bar{m})^{s}(m \bar{p})^{\tilde{j}-s}$ of the $\left(\mathbb{I}^{13}\right)^{\tilde{\jmath}}$ tensor of each structure all contribute to the same term $(p \bar{m})^{s}(m \bar{p})^{j-s} \cdot{ }^{26}$ Concretely we find

$$
\begin{aligned}
\mathcal{A}_{s}\left[\mathrm{t}_{\bar{O} T O}\right]=-\frac{3 i \pi}{4} \int_{\mathbb{R}^{4}} \mathrm{~d}^{4} x e^{-i x^{0}} \sum_{r=0}\left(x^{-}\right)^{s-r-5}\left(x^{+}\right)^{j-r-s-2}\left(x_{\perp}^{2}\right)^{r}\left(x^{2}\right)^{1-\Delta-j / 2} \\
\times\left(I_{r, s}^{(j)}\left(x^{-}\right)^{2}\left(x^{+}\right)^{2} D_{1}-I_{r, s}^{(j-1)} x^{-} x^{+} x^{2} D_{2}+I_{r, s}^{(j-2)}\left(x^{2}\right)^{2} D_{3}\right),
\end{aligned}
$$

where

$$
I_{r, s}^{(j)}=\left(\begin{array}{c}
j \\
2 r
\end{array}\right)\left(\begin{array}{c}
j-2 r \\
s-r
\end{array}\right)\left(\begin{array}{c}
2 r \\
r
\end{array}\right)
$$

\footnotetext{
${ }^{25}$ This statement holds in the $y^{+} \rightarrow \infty$ limit.

${ }^{26}$ To be more precise there are contributions also to the terms $(p \bar{m})^{s+a}(m \bar{p})^{j-s-a}(a=1,2)$, but it can be verified that in the limit $y^{+} \rightarrow \infty$ they are subleading.
} 
Similarly, the denominator has the form

$$
\mathcal{F}_{s}\left[\mathrm{n}_{\bar{O} O}\right]=i^{j} \int_{\mathbb{R}^{4}} \mathrm{~d}^{4} x e^{-i x^{0}} \sum_{r^{\prime}=0} I_{r^{\prime}, s}^{(j)}\left(x^{-}\right)^{s-r^{\prime}}\left(x^{+}\right)^{j-r^{\prime}-s}\left(x_{\perp}^{2}\right)^{r^{\prime}}\left(x^{2}\right)^{-\Delta-j / 2} .
$$

The Fourier transforms can be straightforwardly computed using the general formulas

$$
\begin{aligned}
\int_{\mathbb{R}^{2}} \mathrm{~d}^{2} x_{\perp}\left(x^{2}\right)^{a}\left(x_{\perp}^{2}\right)^{b} & =\frac{\pi \Gamma(1-a-b) \Gamma(1+b)}{\Gamma(-a)}\left(-x^{-} x^{+}\right)^{1+a+b}, \\
\int_{\mathbb{R}^{2}} \mathrm{~d} x^{+} \mathrm{d} x^{-} e^{-i\left(x^{+}+x^{-}\right) / 2}\left(x^{+}\right)^{a}\left(x^{-}\right)^{b} & =\frac{(2 \pi)^{2}(-i)^{a+b}(-2)^{a+b+2}}{\Gamma(-a) \Gamma(-b)} .
\end{aligned}
$$

What remains now is to compute the sums in $r$ and $r^{\prime}$. After some simplifications all sums can be reduced to the following general form for some $m, n:^{27}$

$$
\Sigma_{m, n}=\sum_{r=0}^{\infty} \frac{(-1)^{r}}{r !} \frac{\Gamma\left(\Delta+\frac{j}{2}-r-m\right)}{\Gamma(1-r+s) \Gamma(j-r-s+n)} .
$$

We stress again that even though the upper limit is $\infty$, there are actually only a finite number of nonzero terms. After using the property

$$
\Gamma(X-r)=(-1)^{r} \frac{\Gamma(X)}{(1-X)_{r}}
$$

of the $\Gamma$ function, we can rewrite this sum in the form of a ${ }_{2} F_{1}$ hypergeometric function evaluated at 1 , for which the explicit expression is known:

$$
\begin{aligned}
\Sigma_{m, n} & =\frac{\Gamma\left(\Delta+\frac{j}{2}-m\right)}{\Gamma(1+s) \Gamma(j-s+n)}{ }_{2} F_{1}\left(-s, 1-j-n+s ; 1-\Delta-\frac{j}{2}+m ; 1\right) \\
& =\frac{\Gamma\left(\Delta+\frac{j}{2}-m\right)}{\Gamma(1+s) \Gamma(j-s+n)} \frac{\Gamma\left(1-\Delta-\frac{j}{2}+m\right) \Gamma\left(\frac{j}{2}+m+n-\Delta\right)}{\Gamma\left(s+1-\Delta-\frac{j}{2}+m\right) \Gamma\left(\frac{j}{2}+m+n-\Delta-s\right)} .
\end{aligned}
$$

The final result will be expressed in terms of ratios $\Sigma_{m, n} / \Sigma_{1,1}$ which are rational functions of $\Delta, j$ and $s$. It is now straightforward to check that it agrees with the general formula (5.9).

\section{B.2 Formula for the $\left(\frac{1}{2} j, \frac{1}{2}\right)$ case}

In order to obtain a formula for this case we mostly need to follow the same steps as in the previous subsection, with some minor modifications. The main difference is that the invariants $\mathbb{I}^{31}, \mathbb{J}_{12}^{3}$ and $\mathbb{J}_{23}^{1}$ can yield contributions with $\mathrm{SO}(2)$ charge \pm 1 in the limit $y^{+} \rightarrow \infty$. By looking at table 24 we see that all tensor structures have at most one of these invariant except for $H_{8}$ which contains two. Since that particular structure is zero in our superspace correlator we will not compute a formula for it. As a consequence we need to

\footnotetext{
${ }^{27}(m, n)$ can be $(1,1),(2,1),(3,0)$ or $(4,-1)$
} 
expand $\left(\mathbb{I}^{13}\right)^{\tilde{\jmath}}$ keeping also terms of charge \pm 1 . This is easily done as follows:

$$
\begin{aligned}
\left(\mathbb{I}^{13}\right)^{\tilde{\jmath}}= & \left(m \bar{p} x^{+}+p \bar{m} x^{-}+m \bar{m} \mathrm{x}_{--}+p \bar{p} \mathrm{x}_{+\dot{+}}\right)^{\tilde{\jmath}} \\
= & \sum_{s=0}^{\tilde{\jmath}} \sum_{r=0}^{\min (s, \tilde{\jmath}-s)}\left(\begin{array}{c}
\tilde{\jmath} \\
2 r
\end{array}\right)\left(\begin{array}{c}
\tilde{\jmath}-2 r \\
s-r
\end{array}\right)\left(\begin{array}{c}
2 r \\
r
\end{array}\right)\left(x^{-}\right)^{s-r}\left(x^{+}\right)^{\tilde{\jmath}-r-s}\left(x_{\perp}^{2}\right)^{r}(p \bar{m})^{s}(m \bar{p})^{\tilde{\jmath}-s} \\
& +\sum_{s=0}^{\tilde{\jmath}-1} \sum_{t=0}^{\min (s, \tilde{\jmath}-s-1)}\left(\begin{array}{c}
\tilde{\jmath} \\
2 t+1
\end{array}\right)\left(\begin{array}{c}
\tilde{\jmath}-2 t-1 \\
s-t
\end{array}\right)\left(\begin{array}{c}
2 t+1 \\
t+1
\end{array}\right)\left(x^{-}\right)^{s-t}\left(x^{+}\right)^{\tilde{\jmath}-t-s-1}\left(x_{\perp}^{2}\right)^{t} \\
& \times\left(m \bar{m} \mathrm{x}_{--}+p \bar{p} \mathrm{x}_{+\dot{+}}\right)(p \bar{m})^{s}(m \bar{p})^{\tilde{\jmath}-s-1} .
\end{aligned}
$$

As before, both sums in $r$ and $t$ can be extended to any range. After taking care of the remaining polarizations and performing the Fourier transform with (B.6) we again end up with sums in the form of (B.7). The result will be a $2 \times 2$ matrix whose entries are ratios of $\Gamma$ functions, which can be reduced to rational functions of $\Delta, j$ and $s$. For the extreme cases $s=0$ and $s=j+1$ one needs to retain only the appropriate entry of this matrix - respectively the upper left and the lower right - and discard the other ones. As an example we show the part of the formula that multiplies the coefficient $\mathrm{H}_{2}$ :

$$
\left.\mathcal{E}[\Delta ;(j, 1) ; s]\right|_{H_{2}}=-\frac{3 \pi(-i)^{j+1}(\delta+1)(\delta+j+2)}{8(\delta+j-s+1)_{3}}\left(\begin{array}{cc}
\frac{\delta+j-s+3}{\delta+j-s+1} & \sqrt{\frac{s(j-s+1)}{(s+\delta)(\delta+j-s+1)}} \\
\sqrt{\frac{s(j-s+1)}{(s+\delta)(\delta+j-s+1)}} & \frac{(\delta+s-1)(\delta+j-s+1)}{(\delta+s)(\delta+j-s+4)}
\end{array}\right),
$$

where now $\delta=\Delta-j / 2-5 / 2$ with $\Delta$ the dimension of the operator of $\operatorname{spin}\left(\frac{1}{2} j, \frac{1}{2}\right)$.

Clearly the same logic can be applied to more general cases $\left(\frac{1}{2} j, \frac{1}{2} \bar{\jmath}\right)$ with $\bar{\jmath}$ fixed and $j$ arbitrary. It suffices to expand like in (B.9) keeping terms with charge up to $\pm u$ where $u$ is the total number of invariants $\mathbb{I}^{31}, \mathbb{J}_{12}^{3}$ and $\mathbb{J}_{23}^{1}$ in the tensor structure under consideration. Then all steps follow in the same way, except that one may get sums more complicated than $\Sigma_{m, n}$. 


\section{Tables}

\section{C.1 Ward identities}

\begin{tabular}{|lllll|}
\hline$C_{i}$ & Structure & $j>1$ & $j=1$ & $j=0$ \\
\hline$C_{1}$ & $\mathbb{J}_{13}^{2}\left(\mathbb{I}^{13}\right)^{j}$ & $-\frac{1}{2} C_{2}+\frac{2 i^{j+1}(q-\bar{q})}{3 \pi^{2}}$ & $-\frac{1}{2} C_{2}-\frac{2(q-\bar{q})}{3 \pi^{2}}$ & $\frac{2 i(q-\bar{q})}{3 \pi^{2}}$ \\
\hline$C_{2}$ & $\mathbb{I}^{23}\left(\mathbb{I}^{13}\right)^{j-1}$ & $C_{2}$ & $C_{2}$ & \\
\hline
\end{tabular}

Table 3. Ward identities of the R-current for the correlator $\langle\bar{O} J O\rangle$ when $O$ has spin $\left(\frac{1}{2} j, 0\right)$.

\begin{tabular}{|c|c|c|c|}
\hline$I_{i} \quad$ Structure & $j>1$ & $j=1$ & $j=0$ \\
\hline$I_{1} \quad \mathbb{J}_{23}^{1} \mathbb{I}^{23} \mathbb{I}^{32}\left(\mathbb{I}^{13}\right)^{j-1}$ & $I_{1}$ & & I \\
\hline$I_{2} \quad \mathbb{J}_{13}^{2} \mathbb{I}^{31}\left(\mathbb{I}^{13}\right)^{j}$ & $I_{2}$ & & \\
\hline$I_{3} \quad \mathbb{I}^{21} \mathbb{I}^{32}\left(\mathbb{I}^{13}\right)^{j}$ & $\begin{array}{l}2 I_{2}+I_{5}-\frac{1}{2}\left(I_{1}+I_{6}+I_{4}\right) \\
-\frac{4 i^{j}(3+2(q-\bar{q}))}{3 \pi^{2}}\end{array}$ & & \\
\hline$I_{4} \quad \mathbb{J}_{23}^{1} \mathbb{J}_{12}^{3} \mathbb{I}^{12} \mathbb{I}^{23}\left(\mathbb{I}^{13}\right)^{j-2}$ & $I_{4}$ & / & / \\
\hline$I_{5} \quad \mathbb{I}^{12} \mathbb{I}^{23} \mathbb{I}^{31}\left(\mathbb{I}^{13}\right)^{j-1}$ & $I_{5}$ & & / \\
\hline$I_{6} \quad \mathbb{J}_{12}^{3} \mathbb{I}^{12} \mathbb{I}^{21}\left(\mathbb{I}^{13}\right)^{j-1}$ & $I_{6}$ & & / \\
\hline
\end{tabular}

Table 4. Ward identities of the R-current for the correlator $\left\langle\bar{O}^{\prime} J O^{\prime}\right\rangle$ when $O^{\prime}$ has spin $\left(\frac{1}{2} j, \frac{1}{2}\right)$, R-charge $\frac{2}{3}(q-\bar{q})+1$ and is assumed to be unit normalized. If $O^{\prime}=\bar{Q} O$ the terms not proportional to $I_{k}$ must be rescaled by $c_{(\bar{Q} O)}$. The unbarred entries in the $j=1,0$ columns are obtained by setting the absent coefficients to zero.

\begin{tabular}{|lllll|}
\hline$D_{i}$ & Structure & $j>1$ & $j=1$ & $j=0$ \\
\hline$D_{1}$ & $\left(\mathbb{J}_{13}^{2}\right)^{2}\left(\mathbb{I}^{13}\right)^{j}$ & $D_{1}$ & $\frac{i(2 \Delta-3)}{3 \pi^{2}}$ & $\frac{2 \Delta}{3 \pi^{2}}$ \\
\hline$D_{2}$ & $\mathbb{I}^{12} \mathbb{I}^{23} \mathbb{J}_{13}^{2}\left(\mathbb{I}^{13}\right)^{j-1}$ & $-6 D_{1}+\frac{4 i^{j}(\Delta-j)}{\pi^{2}}$ & $\frac{2 i}{\pi^{2}}$ & $\nearrow$ \\
\cline { 1 - 4 }$D_{3}$ & $\left(\mathbb{I}^{12}\right)^{2}\left(\mathbb{I}^{23}\right)^{2}\left(\mathbb{I}^{13}\right)^{j-2}$ & $6 D_{1}-\frac{2 i^{j}(2 \Delta-3 j)}{\pi^{2}}$ & & \\
\hline
\end{tabular}

Table 5. Ward identities of the stress tensor for the correlator $\langle\bar{O} T O\rangle$ when $O$ has spin $\left(\frac{1}{2} j, 0\right)$. We have defined $\Delta=q+\bar{q}$. 


\begin{tabular}{|c|c|c|c|c|}
\hline$H_{i}$ & Structure & $j>1$ & $j=1$ & $j=0$ \\
\hline$H_{1}$ & $\mathbb{I}^{23} \mathbb{I}^{32} \mathbb{J}_{13}^{2} \mathbb{J}_{23}^{1}\left(\mathbb{I}^{13}\right)^{j-1}$ & $H_{6}$ & $H_{6}$ & $\zeta$ \\
\hline$H_{2}$ & $\mathbb{I}^{31}\left(\mathbb{J}_{13}^{2}\right)^{2}\left(\mathbb{I}^{13}\right)^{j}$ & $\begin{array}{l}-\frac{1}{9}\left(3 H_{5}+2 H_{6}+H_{10}\right) \\
-\frac{1}{6} H_{9}-i^{j+1} \frac{2 \Delta-j-2}{3 \pi^{2}}\end{array}$ & $-\frac{2}{3} H_{6}+\frac{2 \Delta-5}{3 \pi^{2}}$ & $-i \frac{2(\Delta-1)}{3 \pi^{2}}$ \\
\hline$H_{3}$ & $\mathbb{I}^{21} \mathbb{I}^{32} \mathbb{J}_{13}^{2}\left(\mathbb{I}^{13}\right)^{j}$ & $\begin{array}{c}-\frac{1}{9}\left(3 H_{5}+8 H_{6}+H_{10}\right) \\
-\frac{1}{3} H_{9}-\frac{2 i^{j+1}(j-3)}{3 \pi^{2}} \\
\end{array}$ & $-\frac{4}{3} H_{6}-\frac{2}{\pi^{2}}$ & $\frac{2 i}{\pi^{2}}$ \\
\hline$H_{4}$ & $\mathbb{I}^{12} \mathbb{I}^{32}\left(\mathbb{I}^{23}\right)^{2} \mathbb{J}_{23}^{1}\left(\mathbb{I}^{13}\right)^{j-2}$ & $H_{10}$ & $\zeta$ & l \\
\hline$H_{5}$ & $\mathbb{I}^{12} \mathbb{I}^{31} \mathbb{I}^{23} \mathbb{J}_{13}^{2}\left(\mathbb{I}^{13}\right)^{j-1}$ & $H_{5}$ & $\frac{4}{3} H_{6}+\frac{2}{\pi^{2}}$ & / \\
\hline$H_{6}$ & $\mathbb{I}^{12} \mathbb{I}^{21} \mathbb{J}_{13}^{2} \mathbb{J}_{12}^{3}\left(\mathbb{I}^{13}\right)^{j-1}$ & $H_{6}$ & $H_{6}$ & 1 \\
\hline$H_{7}$ & $\mathbb{I}^{12} \mathbb{I}^{21} \mathbb{I}^{23} \mathbb{I}^{32}\left(\mathbb{I}^{13}\right)^{j-1}$ & $\frac{2}{3}\left(H_{6}-H_{10}\right)$ & $\frac{2}{3} H_{6}$ & / \\
\hline$H_{8}$ & $\left(\mathbb{I}^{12}\right)^{2}\left(\mathbb{I}^{23}\right)^{2} \mathbb{J}_{12}^{3} \mathbb{J}_{23}^{1}\left(\mathbb{I}^{13}\right)^{j-3}$ & $\begin{array}{l}-\frac{4}{3}\left(2 H_{6}+H_{10}\right) \\
+2\left(H_{5}+H_{9}\right)+\frac{4 i^{j+1} j}{\pi^{2}}\end{array}$ & / & I \\
\hline$H_{9}$ & $\left(\mathbb{I}^{12}\right)^{2}\left(\mathbb{I}^{23}\right)^{2} \mathbb{I}^{31}\left(\mathbb{I}^{13}\right)^{j-2}$ & $H_{9}$ & 1 & 1 \\
\hline$H_{10}$ & $\left(\mathbb{I}^{12}\right)^{2} \mathbb{I}^{21} \mathbb{I}^{23} \mathbb{J}_{12}^{3}\left(\mathbb{I}^{13}\right)^{j-2}$ & $H_{10}$ & 1 & 1 \\
\hline
\end{tabular}

Table 6. Ward identities of the stress tensor for the correlator $\left\langle\bar{O}^{\prime} T O^{\prime}\right\rangle$ when $O^{\prime}$ has spin $\left(\frac{1}{2} j, \frac{1}{2}\right)$, dimension $\Delta+\frac{1}{2}$ and is assumed to be unit normalized. If $O^{\prime}=\bar{Q} O$ the terms not proportional to $H_{k}$ must be rescaled by $c_{(\bar{Q} O)}$.

\begin{tabular}{|lllll|}
\hline$G_{i}$ & Structure & $j>1$ & $j=1$ & $j=0$ \\
\hline$G_{1}$ & $\mathbb{J}_{23}^{1} \mathbb{J}_{13}^{2} \mathbb{I}^{23}\left(\mathbb{I}^{13}\right)^{j-1}$ & $G_{1}$ & $G_{1}$ & $\nearrow$ \\
\hline$G_{2}$ & $\mathbb{I}^{21} \mathbb{J}_{13}^{2}\left(\mathbb{I}^{13}\right)^{j}$ & $-\frac{1}{2}\left(G_{1}+G_{4}\right)-\frac{1}{3} G_{3}+\frac{8 i^{j} \bar{q}}{3 \pi^{2}}$ & $-\frac{1}{2}\left(G_{1}+G_{4}\right)+\frac{8 i \bar{q}}{3 \pi^{2}}$ & $\frac{8 \bar{q}}{3 \pi^{2}}$ \\
\hline$G_{3}$ & $\mathbb{I}^{12}\left(\mathbb{I}^{23}\right)^{2} \mathbb{J}_{23}^{1}\left(\mathbb{I}^{13}\right)^{j-2}$ & $G_{3}$ & $\nearrow$ & $\nearrow$ \\
\hline$G_{4}$ & $\mathbb{I}^{12} \mathbb{I}^{21} \mathbb{I}^{23}\left(\mathbb{I}^{13}\right)^{j-1}$ & $G_{4}$ & $G_{4}$ & $\nearrow$ \\
\hline
\end{tabular}

Table 7. Ward identities of the supersymmetry current for the correlator $\langle(Q \bar{O}) \bar{S} O\rangle$ when $Q \bar{O}$ has $\operatorname{spin}\left(\frac{1}{2}, \frac{1}{2} j\right)$.

\begin{tabular}{|lllll|}
\hline$E_{i}$ & Structure & $j>1$ & $j=1$ & $j=0$ \\
\hline$E_{1}$ & $\mathbb{J}_{13}^{2} \mathbb{I}^{12}\left(\mathbb{I}^{13}\right)^{j}$ & $-\frac{2}{3} E_{2}-\frac{4 i^{j}(2 q+j)}{3 \pi^{2}(j+1)}$ & $-\frac{2}{3} E_{2}-\frac{2 i(2 q+1)}{3 \pi^{2}}$ & $-\frac{8 q}{3 \pi^{2}}$ \\
\hline$E_{2}$ & $\left(\mathbb{I}^{12}\right)^{2}\left(\mathbb{I}^{13}\right)^{j-1}$ & $E_{2}$ & $E_{2}$ & \\
\hline
\end{tabular}

Table 8. Ward identities of the supersymmetry current for the correlator $\langle(\bar{Q} \bar{O}) S O\rangle$ when $\bar{Q} \bar{O}$ has $\operatorname{spin}\left(0, \frac{1}{2}(j+1)\right)$. 


\begin{tabular}{|llll|}
\hline$F_{i}$ & Structure & $j>1$ & $j=1$ \\
\hline$F_{1}$ & $\mathbb{J}_{13}^{2} \mathbb{K}_{1}^{23}\left(\mathbb{I}^{13}\right)^{j-1}$ & $-\frac{1}{3} F_{2}+\frac{4 i^{j}(2 q-j-2)}{3 \pi^{2}}$ & $\frac{4 i(2 q-3)}{3 \pi^{2}}$ \\
\hline$F_{2}$ & $\mathbb{I}^{12} \mathbb{I}^{23} \mathbb{K}_{1}^{23}\left(\mathbb{I}^{13}\right)^{j-2}$ & $F_{2}$ & $/$ \\
\hline
\end{tabular}

Table 9. Ward identities of the supersymmetry current for the correlator $\langle(\bar{Q} \bar{O}) S O\rangle$ when $\bar{Q} \bar{O}$ has spin $\left(0, \frac{1}{2}(j-1)\right)$.

\section{C.2 Expansion in components}

\begin{tabular}{|llll|}
\hline$C_{i}$ & Structure & $j>0$ & $j=0$ \\
\hline$C_{1}$ & $\mathbb{J}_{13}^{2}\left(\mathbb{I}^{13}\right)^{j}$ & $i\left(\mathcal{C}_{1}+\mathcal{C}_{2}\right)$ & $i \mathcal{C}_{1}$ \\
\hline$C_{2}$ & $\mathbb{I}^{23}\left(\mathbb{I}^{13}\right)^{j-1}$ & $-i \mathcal{C}_{2}$ & $\nearrow$ \\
\hline
\end{tabular}

Table 10. Expansion of the supersymmetric correlator in the component $\langle\bar{O} J O\rangle$ when $O$ has $\operatorname{spin}\left(\frac{1}{2} j, 0\right)$.

\begin{tabular}{|lllll|}
\hline$D_{i}$ & Structure & $j>1$ & $j=1$ & $j=0$ \\
\hline$D_{1}$ & $\left(\mathbb{J}_{13}^{2}\right)^{2}\left(\mathbb{I}^{13}\right)^{j}$ & $-\frac{1}{4}\left(\mathcal{C}_{5}+\mathcal{C}_{8}\right)$ & $-\frac{1}{4}\left(\mathcal{C}_{5}+\mathcal{C}_{8}\right)$ & $-\frac{1}{4} \mathcal{C}_{5}$ \\
\hline$D_{2}$ & $\mathbb{I}^{12} \mathbb{I}^{23} \mathbb{J}_{13}^{2}\left(\mathbb{I}^{13}\right)^{j-1}$ & $\frac{1}{4}\left(\mathcal{C}_{6}+\mathcal{C}_{8}\right)$ & $\frac{1}{4} \mathcal{C}_{8}$ & $\nearrow$ \\
\hline$D_{3}$ & $\left(\mathbb{I}^{12}\right)^{2}\left(\mathbb{I}^{23}\right)^{2}\left(\mathbb{I}^{13}\right)^{j-2}$ & $-\frac{1}{4} \mathcal{C}_{6}$ & $\nearrow$ & \\
\hline
\end{tabular}

Table 11. Expansion of the supersymmetric correlator in the component $\langle\bar{O} T O\rangle$ when $O$ has $\operatorname{spin}\left(\frac{1}{2} j, 0\right)$.

\begin{tabular}{|llll|}
\hline$E_{i}$ & Structure & $j>0$ & $j=0$ \\
\hline$E_{1}$ & $\mathbb{J}_{13}^{2} \mathbb{I}^{12}\left(\mathbb{I}^{13}\right)^{j}$ & $-\frac{1}{2(1+j)}\left(4 \mathcal{C}_{1}+4 \mathcal{C}_{2}+\mathcal{C}_{3}-\mathcal{C}_{5}+\mathcal{C}_{6}-\mathcal{C}_{8}\right)$ & $-2 \mathcal{C}_{1}-\frac{1}{2} \mathcal{C}_{5}$ \\
\hline$E_{2}$ & $\left(\mathbb{I}^{12}\right)^{2}\left(\mathbb{I}^{13}\right)^{j-1}$ & $\frac{1}{2(1+j)}\left(4 \mathcal{C}_{2}+\mathcal{C}_{3}+\mathcal{C}_{6}-\mathcal{C}_{8}\right)$ & \\
\hline
\end{tabular}

Table 12. Expansion of the supersymmetric correlator in the component $\langle(\bar{Q} \bar{O}) S O\rangle$ when $\bar{Q} \bar{O}$ has spin $\left(0, \frac{1}{2}(j+1)\right)$. The result for $j=1$ is obtained by setting $\mathcal{C}_{6}=0$.

\begin{tabular}{|llll|}
\hline$F_{i}$ & Structure & $j>1$ & $j=1$ \\
\hline$F_{1}$ & $\mathbb{J}_{13}^{2} \mathbb{K}_{1}^{23}\left(\mathbb{I}^{13}\right)^{j-1}$ & $2\left(\mathcal{C}_{1}+\mathcal{C}_{2}\right)-\frac{1}{2}\left(\mathcal{C}_{5}+\mathcal{C}_{8}\right)-\frac{1}{2 j}\left(\mathcal{C}_{3}+\mathcal{C}_{6}\right)$ & \\
\hline$F_{2}$ & $\mathbb{I}^{12} \mathbb{I}^{23} \mathbb{K}_{1}^{23}\left(\mathbb{I}^{13}\right)^{j-2}$ & $\frac{1}{j} \mathcal{C}_{6}-\frac{j-1}{2 j}\left(4 \mathcal{C}_{2}+\mathcal{C}_{3}-\mathcal{C}_{8}\right)$ & \\
\hline
\end{tabular}

Table 13. Expansion of the supersymmetric correlator in the component $\langle(\bar{Q} \bar{O}) S O\rangle$ when $\bar{Q} \bar{O}$ has spin $\left(0, \frac{1}{2}(j-1)\right)$. The unbarred entry in the $j=1$ column is obtained by setting $\mathcal{C}_{6}=0$. 


\begin{tabular}{|c|c|c|c|c|}
\hline$G_{i}$ & Structure & $j>1$ & $j=1$ & $j=0$ \\
\hline$G_{1}$ & $\mathbb{J}_{23}^{1} \mathbb{J}_{13}^{2} \mathbb{I}^{23}\left(\mathbb{I}^{13}\right)^{j-1}$ & $\mathcal{C}_{2}-\frac{1}{2} \mathcal{C}_{7}$ & & ／ \\
\hline & $\mathbb{I}^{21} \mathbb{J}_{13}^{2}\left(\mathbb{I}^{13}\right)^{j}$ & $-2\left(\mathcal{C}_{1}+\mathcal{C}_{2}\right)-\frac{1}{2}\left(\mathcal{C}_{5}+\mathcal{C}_{8}\right)$ & & $-2 \mathcal{C}_{1}-\frac{1}{2} \mathcal{C}_{5}$ \\
\hline & $\mathbb{I}^{12}\left(\mathbb{I}^{23}\right)^{2} \mathbb{J}_{23}^{1}\left(\mathbb{I}^{13}\right)^{j-2}$ & $-\frac{1}{2} \mathcal{C}_{6}$ & ～ & l \\
\hline$G_{4}$ & $\mathbb{I}^{12} \mathbb{I}^{21} \mathbb{I}^{23}\left(\mathbb{I}^{13}\right)^{j-1}$ & $\mathcal{C}_{2}+\frac{1}{2}\left(\mathcal{C}_{6}+\mathcal{C}_{7}+\mathcal{C}_{8}\right)$ & & l \\
\hline
\end{tabular}

Table 14. Expansion of the supersymmetric correlator in the component $\langle(Q \bar{O}) \bar{S} O\rangle$ when $Q \bar{O}$ has spin $\left(\frac{1}{2}, \frac{1}{2} j\right)$. The unbarred entries in the $j=1$ column can be obtained by setting the $\mathcal{C}_{6}=0$.

\begin{tabular}{|lll|}
\hline$N_{i}$ & Structure & $j \geqslant 0$ \\
\hline$N_{1}$ & $\mathbb{J}_{13}^{2}\left(\mathbb{I}^{13}\right)^{j+1}$ & $-\frac{2(2 q+j-1)}{(j+1)^{2}}\left(\mathcal{C}_{1}+\mathcal{C}_{2}\right)-\frac{1}{(j+1)^{2}}\left(\mathcal{C}_{4}+\mathcal{C}_{5}+\mathcal{C}_{7}+\mathcal{C}_{8}\right)$ \\
\hline$N_{2}$ & $\mathbb{I}^{12} \mathbb{I}^{23}\left(\mathbb{I}^{13}\right)^{j}$ & $\frac{1}{(j+1)^{2}}\left(2 \mathcal{C}_{1}+2(2 q+j-1) \mathcal{C}_{2}+\mathcal{C}_{4}+\mathcal{C}_{7}+\mathcal{C}_{8}\right)$ \\
\hline
\end{tabular}

Table 15. Expansion of the supersymmetric correlator in the component $\langle(\bar{Q} \bar{O}) J(Q O)\rangle$ when $\bar{Q} \bar{O}$ has spin $\left(0, \frac{1}{2}(j+1)\right)$ and $Q O$ has spin $\left(\frac{1}{2}(j+1), 0\right)$. The result for $j=0,1$ can be obtained by setting the absent coefficients to zero (see caption of table 2 ).

\begin{tabular}{|lll|}
\hline$O_{i}$ & Structure & $j \geqslant 1$ \\
\hline$O_{1}$ & $\mathbb{I}^{12} \overline{\mathbb{K}}_{3}^{12}\left(\mathbb{I}^{13}\right)^{j-1}$ & $\frac{1}{j(j+1)}\left(4(q-1) \mathcal{C}_{2}+\mathcal{C}_{8}\right)-\frac{1}{j+1}\left(2 \mathcal{C}_{1}+\mathcal{C}_{4}+\mathcal{C}_{7}\right)-\frac{1}{j}\left(\mathcal{C}_{3}+\mathcal{C}_{6}\right)$ \\
\hline
\end{tabular}

Table 16. Expansion of the supersymmetric correlator in the component $\langle(\bar{Q} \bar{O}) J(Q O)\rangle$ when $\bar{Q} \bar{O}$ has spin $\left(0, \frac{1}{2}(j+1)\right)$ and $Q O$ has spin $\left(\frac{1}{2}(j-1), 0\right)$. The result for $j=1$ can be obtained by setting $\mathcal{C}_{6}$ to zero.

\begin{tabular}{|lll|}
\hline$P_{i}$ & Structure & $j \geqslant 1$ \\
\hline$P_{1}$ & $\mathbb{I}^{23} \mathbb{K}_{1}^{23}\left(\mathbb{I}^{13}\right)^{j-1}$ & $\frac{1}{j(j+1)}\left(2(2 q-j-3) \mathcal{C}_{2}+\mathcal{C}_{8}+\mathcal{C}_{7}\right)-\frac{1}{j+1}\left(2 \mathcal{C}_{1}+\mathcal{C}_{4}\right)$ \\
\hline
\end{tabular}

Table 17. Expansion of the supersymmetric correlator in the component $\langle(\bar{Q} \bar{O}) J(Q O)\rangle$ when $\bar{Q} \bar{O}$ has spin $\left(0, \frac{1}{2}(j-1)\right)$ and $Q O$ has spin $\left(\frac{1}{2}(j+1), 0\right)$. 


\begin{tabular}{|lll|}
\hline$Q_{i}$ & Structure & $j \geqslant 1$ \\
\hline$Q_{1}$ & $\mathbb{J}_{13}^{2}\left(\mathbb{I}^{13}\right)^{j-1}$ & $-\frac{2 \Xi_{1}}{j} \mathcal{C}_{1}-\frac{2 \Xi_{2}}{j^{2}} \mathcal{C}_{2}-\frac{1}{j^{2}} \mathcal{C}_{7}+\frac{1}{j} \mathcal{C}_{4}+\frac{j+1}{j^{2}}\left(\mathcal{C}_{3}+\mathcal{C}_{6}+j \mathcal{C}_{5}\right)+\frac{j^{2}+j-1}{j^{2}} \mathcal{C}_{8}$ \\
\hline & $\begin{aligned} \frac{2(j-1) \Xi_{3}}{Q_{2}} \mathcal{C}_{2}+\frac{j-1}{j}\left(2 \mathcal{C}_{1}+\mathcal{C}_{4}\right)+\frac{j^{2}-1}{j^{2}} \mathcal{C}_{3}-\frac{2(j+1)}{j^{2}} \mathcal{C}_{6}-\frac{j-1}{j^{2}} \mathcal{C}_{7} \\
\left.\mathbb{I}^{13}\right)^{j-2} \\
\end{aligned}$ \\
& $-\frac{(j-1)(j+2)}{j^{2}} \mathcal{C}_{8}$
\end{tabular}

Table 18. Expansion of the supersymmetric correlator in the component $\langle(\bar{Q} \bar{O}) J(Q O)\rangle$ when $\bar{Q} \bar{O}$ has spin $\left(0, \frac{1}{2}(j-1)\right)$ and $Q O$ has spin $\left(\frac{1}{2}(j-1), 0\right)$. The result for $j=1$ can be obtained by setting $\mathcal{C}_{6}$ to zero and removing the last row. Furthermore we defined

$$
\begin{aligned}
& \Xi_{1}=j^{2}-2 j q+5 j-2 q+3, \\
& \Xi_{2}=j^{3}-2 j^{2} q+5 j^{2}-2 j q+3 j+2 q-3, \\
& \Xi_{3}=j^{2}-2 j q+6 j-4 q+7 .
\end{aligned}
$$

\begin{tabular}{|c|c|c|c|}
\hline $\begin{array}{ll}I_{i} & \text { Structure } \\
\end{array}$ & $j>1$ & $j=1$ & $j=0$ \\
\hline$I_{1} \quad \mathbb{J}_{23}^{1} \mathbb{I}^{23} \mathbb{I}^{32}\left(\mathbb{I}^{13}\right)^{j-1}$ & $\mathcal{C}_{7}-2 \mathcal{C}_{2}$ & & / \\
\hline$I_{2} \quad \mathbb{J}_{13}^{2} \mathbb{I}^{31}\left(\mathbb{I}^{13}\right)^{j}$ & $\begin{array}{l}2(2 \bar{q}-1)\left(\mathcal{C}_{1}+\mathcal{C}_{2}\right) \\
-\mathcal{C}_{4}-\mathcal{C}_{5}-\mathcal{C}_{7}-\mathcal{C}_{8}\end{array}$ & & $2(2 \bar{q}-1) \mathcal{C}_{1}-\mathcal{C}_{4}-\mathcal{C}_{5}$ \\
\hline$I_{3} \quad \mathbb{I}^{21} \mathbb{I}^{32}\left(\mathbb{I}^{13}\right)^{j}$ & $2\left(\mathcal{C}_{1}+\mathcal{C}_{2}\right)-\mathcal{C}_{4}-\mathcal{C}_{7}$ & & $2 \mathcal{C}_{1}-\mathcal{C}_{4}$ \\
\hline$I_{4} \quad \mathbb{J}_{23}^{1} \mathbb{J}_{12}^{3} \mathbb{I}^{12} \mathbb{I}^{23}\left(\mathbb{I}^{13}\right)^{j-2}$ & $\mathcal{C}_{6}$ & / & / \\
\hline$I_{5} \quad \mathbb{I}^{12} \mathbb{I}^{23} \mathbb{I}^{31}\left(\mathbb{I}^{13}\right)^{j-1}$ & $-2(2 \bar{q}-1) \mathcal{C}_{2}-\mathcal{C}_{3}+\mathcal{C}_{7}+\mathcal{C}_{8}$ & & / \\
\hline$I_{6} \quad \mathbb{J}_{12}^{3} \mathbb{I}^{12} \mathbb{I}^{21}\left(\mathbb{I}^{13}\right)^{j-1}$ & $-\mathcal{C}_{3}-\mathcal{C}_{6}$ & & $/$ \\
\hline
\end{tabular}

Table 19. Expansion of the supersymmetric correlator in the component $\langle(Q \bar{O}) J(\bar{Q} O)\rangle$ when $Q \bar{O}$ has spin $\left(\frac{1}{2}, \frac{1}{2} j\right)$. The unbarred entries in the $j=1$ column can be obtained by setting $\mathcal{C}_{6}=0$.

\begin{tabular}{|llll|}
\hline$J_{i}$ & Structure & $j>0$ & $j=0$ \\
\hline$J_{1}$ & $\left(\mathbb{J}_{13}^{2}\right)^{2}\left(\mathbb{I}^{13}\right)^{j+1}$ & $-\frac{i}{2(j+1)^{2}}\left(4 \mathcal{C}_{1}+(2 q+j)\left(\mathcal{C}_{3}+\mathcal{C}_{6}\right)\right.$ & $-2 i \mathcal{C}_{1}+i(2 q-1) \mathcal{C}_{4}$ \\
& & $\left.-(2 q+j-2)\left(4 \mathcal{C}_{2}+2 \mathcal{C}_{4}\right)\right)$ & \\
\hline & & $\frac{i}{(j+1)^{2}}\left(6 \mathcal{C}_{1}+3 \mathcal{C}_{4}-2(2 q+j-10) \mathcal{C}_{2}\right.$ \\
$J_{2}$ & $\mathbb{J}_{13}^{2} \mathbb{I}^{12} \mathbb{I}^{23}\left(\mathbb{I}^{13}\right)^{j}$ & $3 i\left(2 \mathcal{C}_{1}+\mathcal{C}_{4}\right)$ \\
& & $\left.+(2 q+j-1) \mathcal{C}_{3}+(2 q+j) \mathcal{C}_{6}\right)$ & \\
\hline$J_{3}$ & $\left(\mathbb{I}^{12}\right)^{2}\left(\mathbb{I}^{23}\right)^{2}\left(\mathbb{I}^{13}\right)^{j-1}$ & $-\frac{i}{2(j+1)^{2}}\left(32 \mathcal{C}_{2}-4 \mathcal{C}_{3}+(2 q+j) \mathcal{C}_{6}\right)$ & \\
\hline
\end{tabular}

Table 20. Expansion of the supersymmetric correlator in the component $\langle(\bar{Q} \bar{O}) T(Q O)\rangle$ when $\bar{Q} \bar{O}$ has spin $\left(0, \frac{1}{2}(j+1)\right)$ and $Q O$ has $\operatorname{spin}\left(\frac{1}{2}(j+1), 0\right)$. The result for $j=1$ can be obtained by setting $\mathcal{C}_{6}=0$. 


\begin{tabular}{|llll|}
\hline$K_{i}$ & Structure & $j>1$ & $j=1$ \\
\hline \multirow{2}{*}{$K_{1}$} & $\overline{\mathbb{K}}_{3}^{12} \mathbb{J}_{13}^{2} \mathbb{I}^{12}\left(\mathbb{I}^{13}\right)^{j-1}$ & $-\frac{3 i}{j+1}\left(2 \mathcal{C}_{1}+\mathcal{C}_{4}\right)+\frac{i}{j(j+1)}\left((2 q+j-1) \mathcal{C}_{3}\right.$ & \\
& & $\left.-(2 q+j) \mathcal{C}_{6}+2(2 q+7 j-4) \mathcal{C}_{2}\right)$ & \\
\hline$K_{2}$ & $\overline{\mathbb{K}}_{3}^{12} \mathbb{I}^{23}\left(\mathbb{I}^{12}\right)^{2}\left(\mathbb{I}^{13}\right)^{j-2}$ & $\frac{i}{j(j+1)}\left(2(j-1)\left(8 \mathcal{C}_{2}-\mathcal{C}_{3}\right)-(2 q+j) \mathcal{C}_{6}\right)$ & \\
\hline
\end{tabular}

Table 21. Expansion of the supersymmetric correlator in the component $\langle(\bar{Q} \bar{O}) T(Q O)\rangle$ when $\bar{Q} \bar{O}$ has spin $\left(0, \frac{1}{2}(j+1)\right)$ and $Q O$ has spin $\left(\frac{1}{2}(j-1), 0\right)$. The unbarred entry in the $j=1$ column can be obtained by setting $\mathcal{C}_{6}=0$.

\begin{tabular}{|llll|}
\hline$L_{i}$ & Structure & $j>1$ & $j=1$ \\
\hline \multirow{2}{*}{$L_{1}$} & $\mathbb{K}_{1}^{23} \mathbb{J}_{13}^{2} \mathbb{I}^{23}\left(\mathbb{I}^{13}\right)^{j-1}$ & $-\frac{3 i}{j+1}\left(2 \mathcal{C}_{1}+\mathcal{C}_{4}\right)+\frac{i}{j(j+1)}\left((2 q+j-1) \mathcal{C}_{3}\right.$ & \\
& & $\left.-(2 q+j) \mathcal{C}_{6}-2(2 q+7 j-4) \mathcal{C}_{2}\right)$ & \\
\hline$L_{2}$ & $\mathbb{K}_{1}^{23} \mathbb{I}^{12}\left(\mathbb{I}^{23}\right)^{2}\left(\mathbb{I}^{13}\right)^{j-2}$ & $\frac{i}{j(j+1)}\left(2(j-1)\left(8 \mathcal{C}_{2}-\mathcal{C}_{3}\right)-(2 q+j) \mathcal{C}_{6}\right)$ & \\
\hline
\end{tabular}

Table 22. Expansion of the supersymmetric correlator in the component $\langle(\bar{Q} \bar{O}) T(Q O)\rangle$ when $\bar{Q} \bar{O}$ has spin $\left(0, \frac{1}{2}(j-1)\right)$ and $Q O$ has spin $\left(\frac{1}{2}(j+1), 0\right)$. The unbarred entry in the $j=1$ column can be obtained by setting $\mathcal{C}_{6}=0$. Note that this table is identical to table 21 .

\begin{tabular}{|c|c|c|c|c|}
\hline$M_{i}$ & Structure & $j>2$ & $j=2$ & $j=1$ \\
\hline$M_{1}$ & $\left(\mathbb{J}_{13}^{2}\right)^{2}\left(\mathbb{I}^{13}\right)^{j-1}$ & $\begin{array}{l}-\frac{2 i(2 j-1)}{j} \mathcal{C}_{1}+\frac{2 i \Xi_{4}}{j^{2}} \mathcal{C}_{2}-\frac{i \Xi_{5}}{2 j^{2}} \mathcal{C}_{3} \\
+\frac{i \Xi_{6}}{j} \mathcal{C}_{4}-\frac{i(j-1)\left(\Xi_{6}-2 q+j-1\right)}{2 j^{2}} \mathcal{C}_{6}\end{array}$ & & \\
\hline$M_{2}$ & $\mathbb{J}_{13}^{2} \mathbb{I}^{12} \mathbb{I}^{23}\left(\mathbb{I}^{13}\right)^{j-2}$ & $\begin{array}{c}\frac{6 i(j-1)}{j} \mathcal{C}_{1}-\frac{2 i(j-1) \Xi_{7}}{j^{2}} \mathcal{C}_{2}+\frac{3 i(j-1)}{j} \mathcal{C}_{4} \\
+\frac{i(j-1)\left(\Xi_{7}+9 j-12\right)}{j^{2}} \mathcal{C}_{3}+\frac{i \Xi_{8}}{j^{2}} \mathcal{C}_{6}\end{array}$ & & / \\
\hline$M_{3}$ & $\left(\mathbb{I}^{12}\right)^{2}\left(\mathbb{I}^{23}\right)^{2}\left(\mathbb{I}^{13}\right)^{j-3}$ & $\begin{array}{l}\frac{2 i(j-1)(j-2)}{j^{2}}\left(\mathcal{C}_{3}-8 \mathcal{C}_{2}\right) \\
-\frac{i(j-2)\left(j^{2}-2 j q+j-6 q+2\right)}{2 j^{2}} \mathcal{C}_{6}\end{array}$ & / & / \\
\hline
\end{tabular}

Table 23. Expansion of the supersymmetric correlator in the component $\langle(\bar{Q} \bar{O}) T(Q O)\rangle$ when $\bar{Q} \bar{O}$ has spin $\left(0, \frac{1}{2}(j-1)\right)$ and $Q O$ has spin $\left(\frac{1}{2}(j-1), 0\right)$. The unbarred entries in the $j=2$ column are identical and the ones in the $j=1$ column are obtained by setting $\mathcal{C}_{6}=0$. We further defined:

$$
\begin{aligned}
& \Xi_{4}=j^{3}-2 j^{2} q-j^{2}-2 j q+5 j+2 q-4, \\
& \Xi_{5}=j^{3}-2 j^{2} q+j^{2}-2 j q+4 q-4, \\
& \Xi_{6}=j^{2}-2 j q+j-2 q+3, \\
& \Xi_{7}=j^{2}-2 j q-8 j-4 q+18, \\
& \Xi_{8}=j^{3}-2 j^{2} q-2 j q+8 q-3 .
\end{aligned}
$$




\begin{tabular}{|c|c|c|c|}
\hline$H_{i}$ & Structure & $j>1$ & $j=1 \quad j=0$ \\
\hline$H_{1}$ & $\mathbb{I}^{23} \mathbb{I}^{32} \mathbb{J}_{13}^{2} \mathbb{J}_{23}^{1}\left(\mathbb{I}^{13}\right)^{j-1}$ & $\frac{3 i}{2}\left(\mathcal{C}_{3}+\mathcal{C}_{6}\right)$ & I \\
\hline$H_{2}$ & $\mathbb{I}^{31}\left(\mathbb{J}_{13}^{2}\right)^{2}\left(\mathbb{I}^{13}\right)^{j}$ & $\begin{array}{l}-2 i\left(\mathcal{C}_{1}+2 \bar{q} \mathcal{C}_{2}\right)+i(\bar{q}-1)\left(\mathcal{C}_{3}+\mathcal{C}_{6}\right) \\
-i(2 \bar{q}-1) \mathcal{C}_{4}\end{array}$ & \\
\hline$H_{3}$ & $\mathbb{I}^{21} \mathbb{I}^{32} \mathbb{J}_{13}^{2}\left(\mathbb{I}^{13}\right)^{j}$ & $-i\left(6 \mathcal{C}_{1}+2 \mathcal{C}_{3}-3 \mathcal{C}_{4}+2 \mathcal{C}_{6}\right)$ & \\
\hline$H_{4}$ & $\mathbb{I}^{12} \mathbb{I}^{32}\left(\mathbb{I}^{23}\right)^{2} \mathbb{J}_{23}^{1}\left(\mathbb{I}^{13}\right)^{j-2}$ & $-\frac{3 i}{2} \mathcal{C}_{6}$ & l \\
\hline$H_{5}$ & $\mathbb{I}^{12} \mathbb{I}^{31} \mathbb{I}^{23} \mathbb{J}_{13}^{2}\left(\mathbb{I}^{13}\right)^{j-1}$ & $\begin{array}{l}4 i \bar{q} \mathcal{C}_{2}-2 i(\bar{q}-1) \mathcal{C}_{3} \\
\quad-i(2 \bar{q}-3) \mathcal{C}_{6}\end{array}$ & \\
\hline$H_{6}$ & $\mathbb{I}^{12} \mathbb{I}^{21} \mathbb{J}_{13}^{2} \mathbb{J}_{12}^{3}\left(\mathbb{I}^{13}\right)^{j-1}$ & $\frac{3 i}{2}\left(\mathcal{C}_{3}+\mathcal{C}_{6}\right)$ & / \\
\hline$H_{7}$ & $\mathbb{I}^{12} \mathbb{I}^{21} \mathbb{I}^{23} \mathbb{I}^{32}\left(\mathbb{I}^{13}\right)^{j-1}$ & $i\left(\mathcal{C}_{3}+2 \mathcal{C}_{6}\right)$ & $\zeta$ \\
\hline$H_{8}$ & $\left(\mathbb{I}^{12}\right)^{2}\left(\mathbb{I}^{23}\right)^{2} \mathbb{J}_{12}^{3} \mathbb{J}_{23}^{1}\left(\mathbb{I}^{13}\right)^{j-3}$ & 0 & / \\
\hline$H_{9}$ & $\left(\mathbb{I}^{12}\right)^{2}\left(\mathbb{I}^{23}\right)^{2} \mathbb{I}^{31}\left(\mathbb{I}^{13}\right)^{j-2}$ & $i(\bar{q}-2) \mathcal{C}_{6}$ & 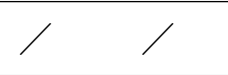 \\
\hline$H_{10}$ & $\left(\mathbb{I}^{12}\right)^{2} \mathbb{I}^{21} \mathbb{I}^{23} \mathbb{J}_{12}^{3}\left(\mathbb{I}^{13}\right)^{j-2}$ & $-\frac{3 i}{2} \mathcal{C}_{6}$ & / \\
\hline
\end{tabular}

Table 24. Expansion of the supersymmetric correlator in the component $\langle(Q \bar{O}) T(\bar{Q} O)\rangle$ when $Q \bar{O}$ has spin $\left(\frac{1}{2}, \frac{1}{2} j\right)$. The unbarred entries in the last two columns can be obtained by setting the absent coefficients to zero (see caption of table 2).

Open Access. This article is distributed under the terms of the Creative Commons Attribution License (CC-BY 4.0), which permits any use, distribution and reproduction in any medium, provided the original author(s) and source are credited.

\section{References}

[1] N.A. Sveshnikov and F.V. Tkachov, Jets and quantum field theory, Phys. Lett. B 382 (1996) 403 [hep-ph/9512370] [INSPIRE].

[2] A.V. Belitsky, G.P. Korchemsky and G.F. Sterman, Energy flow in QCD and event shape functions, Phys. Lett. B 515 (2001) 297 [hep-ph/0106308] [INSPIRE].

[3] C. Lee and G.F. Sterman, Universality of nonperturbative effects in event shapes, eConf $\mathbf{C}$ 0601121 (2006) A001 [hep-ph/0603066] [INSPIRE].

[4] D.M. Hofman and J. Maldacena, Conformal collider physics: energy and charge correlations, JHEP 05 (2008) 012 [arXiv:0803.1467] [INSPIRE].

[5] T. Faulkner, R.G. Leigh, O. Parrikar and H. Wang, Modular Hamiltonians for deformed half-spaces and the averaged null energy condition, JHEP 09 (2016) 038 [arXiv: 1605.08072] [INSPIRE].

[6] T. Hartman, S. Kundu and A. Tajdini, Averaged null energy condition from causality, JHEP 07 (2017) 066 [arXiv: 1610.05308] [INSPIRE]. 
[7] Z. Komargodski, M. Kulaxizi, A. Parnachev and A. Zhiboedov, Conformal field theories and deep inelastic scattering, Phys. Rev. D 95 (2017) 065011 [arXiv:1601.05453] [InSPIRE].

[8] D. Meltzer, Higher spin ANEC and the space of CFTs, JHEP 07 (2019) 001 [arXiv: 1811.01913] [INSPIRE].

[9] P. Kravchuk and D. Simmons-Duffin, Light-ray operators in conformal field theory, JHEP 11 (2018) 102 [arXiv: 1805.00098] [INSPIRE].

[10] C. Cordova and K. Diab, Universal bounds on operator dimensions from the average null energy condition, JHEP 02 (2018) 131 [arXiv:1712.01089] [INSPIRE].

[11] G. Mack, All unitary ray representations of the conformal group $\mathrm{SU}(2,2)$ with positive energy, Commun. Math. Phys. 55 (1977) 1 [InSPIRE].

[12] B. Grinstein, K.A. Intriligator and I.Z. Rothstein, Comments on unparticles, Phys. Lett. B 662 (2008) 367 [arXiv:0801.1140] [INSPIRE].

[13] M. Flato and C. Fronsdal, Representations of conformal supersymmetry, Lett. Math. Phys. 8 (1984) 159 [INSPIRE].

[14] V.K. Dobrev and V.B. Petkova, All positive energy unitary irreducible representations of extended conformal supersymmetry, Phys. Lett. 162B (1985) 127 [INSPIRE].

[15] S. Minwalla, Restrictions imposed by superconformal invariance on quantum field theories, Adv. Theor. Math. Phys. 2 (1998) 783 [hep-th/9712074] [INSPIRE].

[16] S. Ferrara and B. Zumino, Transformation properties of the supercurrent, Nucl. Phys. B 87 (1975) 207 [INSPIRE].

[17] J.-H. Park, $N=1$ superconformal symmetry in four-dimensions, Int. J. Mod. Phys. A 13 (1998) 1743 [hep-th/9703191] [INSPIRE].

[18] H. Osborn, $N=1$ superconformal symmetry in four-dimensional quantum field theory, Annals Phys. 272 (1999) 243 [hep-th/9808041] [INSPIRE].

[19] C. Cordova, T.T. Dumitrescu and K. Intriligator, Multiplets of superconformal symmetry in diverse dimensions, JHEP 03 (2019) 163 [arXiv: 1612.00809] [INSPIRE].

[20] A. Ceresole, G. Dall'Agata, R. D'Auria and S. Ferrara, Spectrum of type IIB supergravity on $A d S_{5} \times T^{1} 1$ : Predictions on $N=1$ SCFT's, Phys. Rev. D 61 (2000) 066001 [hep-th/9905226] [INSPIRE].

[21] F. Cachazo, M.R. Douglas, N. Seiberg and E. Witten, Chiral rings and anomalies in supersymmetric gauge theory, JHEP 12 (2002) 071 [hep-th/0211170] [INSPIRE].

[22] M. Buican, T. Nishinaka and C. Papageorgakis, Constraints on chiral operators in $\mathcal{N}=2$ SCFTs, JHEP 12 (2014) 095 [arXiv:1407.2835] [INSPIRE].

[23] D. Simmons-Duffin, A semidefinite program solver for the conformal bootstrap, JHEP 06 (2015) 174 [arXiv: 1502.02033] [INSPIRE].

[24] J. Wess and J. Bagger, Supersymmetry and supergravity, Princeton University Press, Princeton U.S.A. (1992).

[25] A. Manenti, A. Stergiou and A. Vichi, R-current three-point functions in $4 d \mathcal{N}=1$ superconformal theories, JHEP 12 (2018) 108 [arXiv:1804.09717] [INSPIRE].

[26] P. Kravchuk and D. Simmons-Duffin, Counting conformal correlators, JHEP 02 (2018) 096 [arXiv: 1612.08987] [INSPIRE]. 
[27] Z. Komargodski and N. Seiberg, Comments on supercurrent multiplets, supersymmetric field theories and supergravity, JHEP 07 (2010) 017 [arXiv: 1002.2228] [INSPIRE].

[28] G.F. Cuomo, D. Karateev and P. Kravchuk, General bootstrap equations in $4 D$ CFTs, JHEP 01 (2018) 130 [arXiv:1705.05401] [INSPIRE].

[29] D. Li and A. Stergiou, Two-point functions of conformal primary operators in $\mathcal{N}=1$ superconformal theories, JHEP 10 (2014) 037 [arXiv: 1407.6354] [INSPIRE].

[30] D. Karateev, P. Kravchuk, M. Serone and A. Vichi, Fermion conformal bootstrap in 4d, JHEP 06 (2019) 088 [arXiv: 1902.05969] [INSPIRE].

[31] A. Zhiboedov, On conformal field theories with extremal a/c values, JHEP 04 (2014) 038 [arXiv:1304.6075] [INSPIRE].

[32] C. Cordova, J. Maldacena and G.J. Turiaci, Bounds on OPE coefficients from interference effects in the conformal collider, JHEP 11 (2017) 032 [arXiv:1710.03199] [INSPIRE].

[33] D. Poland, S. Rychkov and A. Vichi, The conformal bootstrap: theory, numerical techniques and applications, Rev. Mod. Phys. 91 (2019) 015002 [arXiv:1805.04405] [INSPIRE].

[34] I. Klep and M. Schweighofer, Infeasibility certificates for linear matrix inequalities, arXiv: 1108.5930.

[35] M. Laurent, Sums of squares, moment matrices and optimization over polynomials, Emerging Appl. Alg. Goem. 149 (2009) 157.

[36] M. Kojima, Sums of squares relaxations of polynomial semidefinite programs, Technical report, Tokyo Institute of Technology, Tokyo Japan (2003).

[37] C.W. Scherer and C.W.J. Hol, Matrix sum-of-squares relaxations for robust semi-definite programs, Math. Prog. 107 (2006) 189.

[38] F.A. Dolan and H. Osborn, On short and semi-short representations for four-dimensional superconformal symmetry, Annals Phys. 307 (2003) 41 [hep-th/0209056] [INSPIRE].

[39] S.M. Kuzenko and S. Theisen, Correlation functions of conserved currents in $N=2$ superconformal theory, Class. Quant. Grav. 17 (2000) 665 [hep-th/9907107] [INSPIRE].

[40] I.A. Ramírez, Mixed OPEs in $\mathcal{N}=2$ superconformal theories, JHEP 05 (2016) 043 [arXiv: 1602.07269] [INSPIRE].

[41] J. Maldacena and A. Zhiboedov, Constraining conformal field theories with a higher spin symmetry, J. Phys. A 46 (2013) 214011 [arXiv:1112.1016] [InSPIRE].

[42] V. Alba and K. Diab, Constraining conformal field theories with a higher spin symmetry in $d>3$ dimensions, JHEP 03 (2016) 044 [arXiv: 1510.02535] [inSPIRE]. 